\title{
OPERATORS IN THE SPACES OF PSEUDOCHARACTERS OF BRAID GROUPS
}

\author{
A. V. MALYUTIN
}

\begin{abstract}
The theory of pseudocharacters of Artin's braid groups is developed. A family of operators in the spaces of pseudocharacters of braid groups is introduced and studied, techniques for constructing new pseudocharacters for braid groups are described, and the structure of the space of pseudocharacters of the braid group is investigated.
\end{abstract}

\section{INTRODUCTION}

0.1. Pseudocharacters. A function $\varphi: G \rightarrow \mathbb{R}$ on a group $G$ is called a quasicharacter (or quasimorphism) with defect $C$ if the following condition is fulfilled:

$$
\sup _{g_{1}, g_{2} \in G}\left|\varphi\left(g_{1} g_{2}\right)-\varphi\left(g_{1}\right)-\varphi\left(g_{2}\right)\right|=C<\infty .
$$

If, moreover, we have

$$
\forall z \in \mathbb{Z}, g \in G: \quad \varphi\left(g^{z}\right)=z \varphi(g),
$$

then $\varphi$ is called a pseudocharacter (or pseudomorphism).

It is known that for each quasicharacter $\psi: G \rightarrow \mathbb{R}$ there is a unique pseudocharacter $\bar{\psi}: G \rightarrow \mathbb{R}$ such that the function $\psi-\bar{\psi}$ is bounded. Furthermore, for each $g \in G$, we have $\bar{\psi}(g)=\lim _{n \rightarrow \infty} \frac{\psi\left(g^{n}\right)}{n}$.

The above definition shows that the pseudocharacters of a group $G$ form a real vector space. We denote this space by $\mathcal{P X}(G)$. Since any homomorphism $G \rightarrow \mathbb{R}$ is a pseudocharacter with defect 0 , the space $\operatorname{Hom}(G, \mathbb{R})$ of real-valued homomorphisms is a subspace of $\mathcal{P X}(G)$. It is well known that each pseudocharacter is conjugacy invariant.

0.2. Pseudocharacters of braid groups. The Artin braid group of infinite rank is determined by the presentation

$$
B_{\infty}:=\left\langle\sigma_{1}, \sigma_{2}, \ldots\left|\sigma_{i} \sigma_{j}=\sigma_{j} \sigma_{i},\right| i-j \mid \geq 2 ; \sigma_{i} \sigma_{i+1} \sigma_{i}=\sigma_{i+1} \sigma_{i} \sigma_{i+1}\right\rangle .
$$

The elements of $B_{\infty}$ are called braids. The generators $\sigma_{i}$ are Artin's generators. The Artin braid group of rank $n$ with $n \geq 2$ is the subgroup of $B_{\infty}$ generated by the elements $\sigma_{1}, \ldots, \sigma_{n-1}$. The group $B_{2}$ is isomorphic to $\mathbb{Z}$. The groups $B_{0}$ and $B_{1}$ are trivial. If $k<n$, then $B_{k}$ is a subgroup of $B_{n}$. We denote the natural inclusion $B_{k} \rightarrow B_{n}$ by $\mathcal{I}_{n, k}$.

If $n \geq 3$, then the space $\mathcal{P X}\left(B_{n}\right)$ is infinite-dimensional. (This fact follows, for instance, from the results of 22.) If $n \geq 2$, then the space $\operatorname{Hom}\left(B_{n}, \mathbb{R}\right)$ is one-dimensional. Since $B_{2} \simeq \mathbb{Z}$, it follows that $\mathcal{P X}\left(B_{2}\right)=\operatorname{Hom}\left(B_{2}, \mathbb{R}\right)$. The space $\mathcal{P} \mathcal{X}\left(B_{\infty}\right)$ also consists

2000 Mathematics Subject Classification. Primary 20F36.

Key words and phrases. Quasicharacter, pseudocharacter, braid group.

Supported in part by RFBR (grant 08-01-00379-a) and by the RF President's Program for Support of Leading Scientific Schools (grant NSh-2460.2008.1112). 
of homomorphisms and has dimension 1 . The homomorphism $B_{n} \rightarrow \mathbb{Z}$ sending $\sigma_{i}$ to 1 is called the exponential sum. The exponential sum is a unique (up to multiplication by a constant) real-valued homomorphism on $B_{n}$. In the literature, there are also descriptions for the following pseudocharacters and quasicharacters of braid groups: the twist number (see [10]); quasicharacters corresponding to the signatures of links represented by braids (see [6]); and the quasicharacter of $B_{3}$ induced by the standard homomorphism $B_{3} \rightarrow \mathbf{S L}(2, \mathbb{Z})$ and the Rademacher function on $\mathbf{S L}(2, \mathbb{Z})$. (The results of $[6$ and 8$]$ imply that the pseudocharacter of $B_{3}$ corresponding to the latter ("Rademacher's") quasicharacter is a linear combination of the twist number and the exponential sum.)

0.3. Initially, our research was motivated by the problem of finding new pseudocharacters for braid groups. This problem is of interest because pseudocharacters of braid groups have found various applications in low-dimensional topology and dynamics (see [5, 6, 7, 8, 10, 11). At the same time, only a few series of braid group pseudocharacters have been described explicitly up to the present time (see the cited papers).

One of the results of the present paper is a technique for constructing new pseudocharacters for braid groups from known ones. More precisely, we give a family of combinatorial formulas that determine mappings in the spaces of functions on braid groups, and we prove that these mappings send pseudocharacters to pseudocharacters. Formulas in our family automatically convert any computational algorithm for a pseudocharacter of the braid group into a computational algorithm for new pseudocharacters.

Our combinatorial formulas are mappings from groups to group rings (this is explained below in detail). The induced mappings of spaces of pseudocharacters are linear operators. These operators are the central object of the present work. We study their basic properties, which allows us to obtain a series of new results concerning pseudocharacters of braid groups and the structure of the space of pseudocharacters of the braid group.

The techniques introduced in the paper provide us with a large set of operators in the spaces of pseudocharacters of braid groups. However, we restrict our study to a relatively small family in the above set. This family consists of the simplest and most natural operators of this kind. The operators of the family are said to be $\Gamma$-diagonal. This is a closed set of operators. In the space of all linear operators $\mathcal{P X}\left(B_{n}\right) \rightarrow \mathcal{P X}\left(B_{k}\right)$ with $n, k \in \mathbb{N}$, the $\Gamma$-diagonal operators form a subspace of dimension $\min (k-1, n-1)$. All $\Gamma$-diagonal operators in $\mathcal{P X}\left(B_{n}\right)$ are diagonal.

To describe our operators, we exploit two approaches. One of them is based upon the geometric representation of braids. This approach is convenient for proving various properties of the operators. Another approach is based upon a general construction of transfer of pseudocharacters. (The transfer of pseudocharacters is a specific operator $\mathcal{P X}(H) \rightarrow \mathcal{P X}(G)$, where $G$ is a group with a normal subgroup $H$ of finite index. It is an analog of the cohomology transfer.) The latter approach plays an auxiliary role in this paper. However, below we discuss it first, because it allows us to prove the key fact that the mappings we consider send pseudocharacters to pseudocharacters.

The transfer of pseudocharacters is introduced in $\S 2$. In $\S 3$, we use the transfer to construct a series of $\Gamma$-diagonal operators $\widetilde{\mathcal{R}}_{n, k}^{!}: \mathcal{P X}\left(B_{k}\right) \rightarrow \mathcal{P X}\left(B_{n}\right)(k<n)$. (The $\Gamma$-diagonal operators are defined in $\S 8$. The $\Gamma$-diagonality of $\widetilde{\mathcal{R}}_{n, k}^{!}$is proved in $\S 9$.) In $\S \S 4-6$, we develop the aforementioned geometric approach to the description of our operators. In $\oiint 4$ and 5, we introduce strand removal procedures for braids. These procedures have a clear geometric interpretation. In $\S 6$, we describe the operator $\widetilde{\mathcal{R}}_{n, k}^{!}$ in terms of strand removal procedures.

In $\S 7$, we construct yet another series of $\Gamma$-diagonal operators

$$
\mathcal{A}_{n, k}^{!}: \mathcal{P X}\left(B_{k}\right) \rightarrow \mathcal{P X}\left(B_{n}\right) \quad(k<n) .
$$


A remarkable property of $\mathcal{A}_{n, k}^{!}$is the relation $\mathcal{I}_{n, k}^{!} \circ \mathcal{A}_{n, k}^{!}=\operatorname{id}_{\mathcal{P X}\left(B_{k}\right)}$, where

$$
\mathcal{I}_{n, k}^{!}: \mathcal{P X}\left(B_{n}\right) \rightarrow \mathcal{P X}\left(B_{k}\right)
$$

is the natural projection that sends pseudocharacters of $B_{n}$ to their restrictions to the subgroup $B_{k} \subset B_{n}$. It follows that $\mathcal{I}_{n, k}^{!}$is a surjection. In other words, the following is true.

Corollary (to Proposition 7.2). Let $0 \leq k<n$. Then each pseudocharacter of the braid group $B_{k}$ extends to a pseudocharacter of the braid group $B_{n} \supset B_{k}$.

It should be mentioned that we prove this fact constructively; i.e., our technique allows us to find a combinatorial formula for $\mathcal{A}_{n, k}^{!}$, which converts an algorithm for a pseudo-character $\phi: B_{k} \rightarrow \mathbb{R}$ into an algorithm for the extension $\mathcal{A}_{n, k}^{!}(\phi): B_{n} \rightarrow \mathbb{R}$.

It is natural to view the operators $\mathcal{A}_{n, k}^{!}$as canonical injections for spaces of pseudocharacters of braid groups. This viewpoint endows the space $\mathcal{P X}\left(B_{n}\right)$ with a certain inner structure. We describe and study this structure in $\S 8$, where the notions of kernel and subkernel pseudocharacters, and also the notions of degree and rank for a pseudocharacter are introduced.

\section{§1. PSEudohomomorphic MAPPINGS AND RING OPERATORS}

1.1. In certain cases, new pseudocharacters can be obtained from known ones by homomorphisms. It is easily seen that the composition $\varphi \circ f: G \rightarrow \mathbb{R}$ of a group homomorphism $f: G \rightarrow H$ and a pseudocharacter $\varphi: H \rightarrow \mathbb{R}$ is a pseudocharacter. In terms of category theory, this means that the functor that sends a group to the space of pseudocharacters of the group is contravariant: a group homomorphism $f: G \rightarrow H$ induces a linear operator $f^{!}: \mathcal{P X}(H) \rightarrow \mathcal{P X}(G)$. For example, the homomorphism from $B_{4}$ to $B_{3}$ defined by $\sigma_{1} \mapsto \sigma_{1}, \sigma_{2} \mapsto \sigma_{2}, \sigma_{3} \mapsto \sigma_{1}$ gives rise to new pseudocharacters for $B_{4}$. It turns out, however, that for the braid groups the above example is one of a few exceptions, and our attempts to obtain new pseudocharacters with the help of homomorphisms between braid groups fail as a rule. The following remarks (a)-(c) illustrate this.

(a) It is clear that any automorphism of a group $G$ induces an automorphism on $\mathcal{P X}(G)$. Since pseudocharacters are conjugacy invariant, each inner automorphism of $G$ induces the identity operator on $\mathcal{P X}(G)$. Therefore, the group $\operatorname{Out}(G)=\operatorname{Aut}(G) / \operatorname{Inn}(G)$ of outer automorphisms acts naturally on $\mathcal{P X}(G)$. It is known that $\operatorname{Out}\left(B_{n}\right) \cong \mathbb{Z} / 2 \mathbb{Z}$ if $n \geq 2$ (see [4]). The operator $\xi^{!}: \mathcal{P X}\left(B_{n}\right) \rightarrow \mathcal{P X}\left(B_{n}\right)$ induced by the nontrivial element $\xi \in \operatorname{Out}\left(B_{n}\right)$ reverses the signs of all known pseudocharacters of braid groups. (A problem: does $\xi^{!}$coincide with $-\operatorname{id}_{\mathcal{P X}\left(B_{n}\right)}$ ?)

(b) If $k<n \neq 4$, then each nontrivial homomorphism $f: B_{n} \rightarrow B_{k}$ has infinite cyclic image (see [9]). This implies that the pseudocharacter $\varphi \circ f: B_{n} \rightarrow \mathbb{R}$ is a homomorphism for every pseudocharacter $\varphi: B_{k} \rightarrow \mathbb{R}$. But $B_{n}$ admits a unique (up to multiplication by a constant) nontrivial real-valued homomorphism.

(c) If $G$ is a group with a subgroup $H$, then the operator $\mathcal{I}^{!}: \mathcal{P X}(G) \rightarrow \mathcal{P X}(H)$ induced by the natural inclusion $\mathcal{I}: H \rightarrow G$ sends a pseudocharacter $\phi: G \rightarrow \mathbb{R}$ to its restriction $\left.\phi\right|_{H}: H \rightarrow \mathbb{R}$. The natural inclusion $\mathcal{I}_{n, k}: B_{k} \rightarrow B_{n}(k<n)$ does not provide us with "new" pseudocharacters, because the known pseudocharacters form several series $\phi_{i} \in \mathcal{P X}\left(B_{i}\right), i=1,2,3, \ldots$, such that either $\phi_{n} \circ \mathcal{I}_{n, k}=\phi_{k}$ or $\phi_{n} \circ \mathcal{I}_{n, k}=0$.

Several other known endomorphisms of $B_{n}$ and homomorphisms $B_{k} \rightarrow B_{n}$ with $k<n$ also do not produce new pseudocharacters. However, it turns out that for braid groups there are natural mappings of group rings that induce injective operators in spaces of pseudocharacters. To describe and study these mappings, we introduce a specific terminology. 
1.2. Pseudohomomorphic mappings and ring operators in spaces of pseudocharacters. An arbitrary map $f: G \rightarrow \mathbb{R} H$ from a group $G$ to the group ring $\mathbb{R} H$ of a group $H$ induces a linear operator $f^{*}$ from the space of functions on $H$ to the space of functions on $G$. We say that $f$ is pseudohomomorphic if the image $f^{*}(\mathcal{P X}(H))$ is in $\mathcal{P X}(G)$. If $f: G \rightarrow \mathbb{R} H$ is pseudohomomorphic, then we call the restriction $\left.f^{*}\right|_{\mathcal{P X}(H)}: \mathcal{P X}(H) \rightarrow \mathcal{P X}(G)$ a ring operator of pseudocharacters and denote it by $f^{!}$. Two pseudohomomorphic maps $f_{1}: G \rightarrow \mathbb{R} H$ and $f_{2}: G \rightarrow \mathbb{R} H$ are said to be equivalent if $f_{1}^{!}=f_{2}^{!}$. We say that a map $f: G \rightarrow \mathbb{R} H$ is homomorphic if it is a linear combination of homomorphisms. (Thus, any homomorphic map is pseudohomomorphic.)

1.3. Convention. In what follows, we identify mappings from groups to group rings with their linear extensions to mappings of group rings. In particular, by the composition $f_{2} \circ f_{1}$ of mappings $f_{1}: F \rightarrow \mathbb{R} G$ and $f_{2}: G \rightarrow \mathbb{R} H$ from groups to group rings we mean the composition $\bar{f}_{2} \circ f_{1}: F \rightarrow \mathbb{R} H$, where $\bar{f}_{2}: \mathbb{R} G \rightarrow \mathbb{R} H$ is the linear extension of $f_{2}$. This does not cause any confusion, because the composition $f_{2} \circ f_{1}$ is not well defined in the usual sense.

We also identify functions on groups (in particular, pseudocharacters) with their linear extensions to group rings. For example, if $\phi: G \rightarrow \mathbb{R}$ is a function and $f: F \rightarrow \mathbb{R} G$ is a mapping, then by $\phi \circ f$ we mean the function $f^{*}(\phi): F \rightarrow \mathbb{R}$, where $f^{*}$ is the linear operator from the space of functions on $G$ to the space of functions on $F$ induced by $f$.

1.4. Remarks. It is easily seen that the compositions and linear combinations (see Convention 1.3) of pseudohomomorphic mappings are pseudohomomorphic. Consequently, the compositions and linear combinations of ring operators are ring operators.

If the space of pseudocharacters $\mathcal{P X}(H)$ of a group $H$ is finite-dimensional, then each linear operator $\mathcal{P X}(H) \rightarrow \mathcal{P X}(G)$ is a ring operator, while if $\mathcal{P X}(H)$ is infinitedimensional and $\mathcal{P X}(G)$ is nontrivial, then there exists an operator $\mathcal{P X}(H) \rightarrow \mathcal{P X}(G)$ that is not a ring operator.

Obviously, the ring operators share some useful properties of operators induced by homomorphisms. In particular, if we have an explicit formula for a pseudohomomorphic mapping $f: G \rightarrow \mathbb{R} H$ and a computational algorithm for a pseudocharacter $\phi: H \rightarrow \mathbb{R}$, then we can easily obtain an algorithm for the pseudocharacter $f^{!}(\phi): G \rightarrow \mathbb{R}$.

In the following section, we discuss the above-mentioned notion of transfer for pseudocharacters. The transfer provides plenty of examples of pseudohomomorphic mappings that are not equivalent to homomorphic ones (note that by combining a pseudohomomorphic mapping with homomorphic mappings we can obtain a large series of pseudohomomorphic mappings that are not equivalent to homomorphic ones).

\section{§2. TRANSFER OF PSEUdochaRACTERS}

The standard cohomology transfer (see, e.g., 3) has an analog for the bounded cohomology and pseudocharacters. The transfer of pseudocharacters is defined as follows. Let $H$ be a normal subgroup of finite index $s$ in a group $G$. Let $Q \subset G$ be a set of representatives of the cosets of $H$ in $G$. We have $g^{s} \in H$ for each $g \in G$. Since $H$ is a normal subgroup, for any $h \in H$ and $g \in G$ we have $g h g^{-1} \in H$. Therefore, the formula

$$
g \mapsto \frac{\sum_{q \in Q} q g^{s} q^{-1}}{s^{2}}
$$

determines a mapping from $G$ to the group ring $\mathbb{R} H$. We denote this mapping by $\mathcal{T}_{G ; H ; Q}$.

2.1. Proposition. The mapping $\mathcal{T}_{G ; H ; Q}$ is pseudohomomorphic. Furthermore, the induced ring operator $\mathcal{T}_{G ; H ; Q}^{!}: \mathcal{P X}(H) \rightarrow \mathcal{P X}(G)$ is uniquely determined by the pair $(G, H)$; 
i.e., it does not depend on the set $Q$. (For this reason, from now on we denote this operator by $\mathcal{T}_{G ; H}^{!}$.)

The ring operator $\mathcal{T}_{G ; H}^{!}$is called the transfer.

2.2. Remark. The transfer operator $\mathcal{T}_{G ; H}^{!}$is surjective, because the proof of Proposition 2.1 (see below) shows that $\mathcal{T}_{G ; H}^{!} \circ \mathcal{J}^{!}=\operatorname{id}_{\mathcal{P X}(G)}$, where $\mathcal{J}^{!}: \mathcal{P X}(G) \rightarrow \mathcal{P X}(H)$ is the operator induced by the natural inclusion $\mathcal{J}: H \rightarrow G$.

2.3. Remark. The coefficient $1 / s^{2}$ in the defining formula for $\mathcal{T}_{G ; H ; Q}$ arises in a natural way. (Multiplication by a nonzero coefficient sends a pseudohomomorphic mapping to a pseudohomomorphic mapping.) The identity $\mathcal{T}_{G ; H}^{!} \circ \mathcal{J}^{!}=\operatorname{id}_{\mathcal{P X}(G)}$ mentioned above indicates that the coefficient is "proper".

The rest of this section is devoted to the proof of Proposition 2.1

Proof of Proposition 2.1. We introduce several auxiliary objects. Let $\mathcal{P}_{G ; H ; Q}: H \rightarrow \mathbb{R} H$ be the mapping defined by

$$
\mathcal{P}_{G ; H ; Q}(h):=\frac{\sum_{q \in Q} q h q^{-1}}{s} .
$$

Let $\Lambda_{s}: G \rightarrow \mathbb{R} H$ be the mapping defined by

$$
\Lambda_{s}(g):=g^{s} / s .
$$

By Convention 1.3 , we have

$$
\mathcal{T}_{G ; H ; Q}=\mathcal{P}_{G ; H ; Q} \circ \Lambda_{s}
$$

Let $\mathcal{P} \mathcal{X}_{G}(H)$ denote the subspace of $\mathcal{P X}(H)$ consisting of the pseudocharacters of $H$ that extend to $G$.

We recall that the action of $G$ on $H$ by conjugation automorphisms induces an action of $G$ on $\mathcal{P X}(H)$. Let $g^{!}$denote the automorphism of $\mathcal{P X}(H)$ that corresponds to conjugation by $g \in G$. (For $g \in G, \phi \in \mathcal{P X}(H)$, and $h \in H$, we have $\left(g^{!}(\phi)\right)(h)=\phi\left(g h g^{-1}\right)$.) We say that a pseudocharacter $\phi \in \mathcal{P X}(H)$ is $G$-invariant if $g^{!}(\phi)=\phi$ for each $g \in G$.

2.4. Claim. A pseudocharacter $\phi: H \rightarrow \mathbb{R}$ is in $\mathcal{P X}_{G}(H)$ if and only if it is $G$-invariant.

Proof. This was proved in [11, Lemma 4.2].

2.5. Claim.

1) The map $\mathcal{P}_{G ; H ; Q}: H \rightarrow \mathbb{R} H$ is homomorphic.

2) The ring operator $\mathcal{P}_{G ; H ; Q}^{!}$does not depend on $Q$.

3) The image $\mathcal{P}_{G ; H ; Q}^{!}(\mathcal{P X}(H))$ lies in $\mathcal{P X}_{G}(H)$.

4) If $\phi \in \mathcal{P X}_{G}(H)$, then $\mathcal{P}_{G ; H ; Q}^{!}(\phi)=\phi$.

Proof of Claim 2.5. 1) Since for each $q \in Q$ the mapping $h \mapsto q h q^{-1}$ is a group automorphism of $H$, the mapping $\mathcal{P}_{G ; H ; Q}$ is a linear combination of automorphisms, i.e., is homomorphic.

2) Let $H_{1}, \ldots, H_{s}$ be the cosets of $H$ in $G$. Since the pseudocharacters are conjugacy invariant, it follows that for each $h \in H$ the automorphism $h^{!}: \mathcal{P X}(H) \rightarrow \mathcal{P X}(H)$ (the notation $h^{!}$was introduced above) is the identity. This means that all elements of a coset $H_{i}$ induce one and the same automorphism $H_{i}^{!}: \mathcal{P X}(H) \rightarrow \mathcal{P X}(H)$. It follows that the operator $\mathcal{P}_{G ; H ; Q}^{!}$coincides with the operator $\left(H_{1}^{!}+\cdots+H_{s}^{!}\right) / s$, which shows that assertion 2) is true.

3) Let $\phi \in \mathcal{P X}(H)$ be an arbitrary pseudocharacter. Set $\phi_{i}:=H_{i}^{!}(\phi)$ for $i \in\{1, \ldots, s\}$. Then $\mathcal{P}_{G ; H ; Q}^{!}(\phi)=\left(\phi_{1}+\cdots+\phi_{s}\right) / s$. We observe that for every $g \in G$, the left shift 
$x \mapsto g x$ on $G$ induces a permutation on the set $\left\{H_{1}, \ldots, H_{s}\right\}$, so that the automorphism $g^{\text {! }}$ acts on $\left\{\phi_{1}, \ldots, \phi_{s}\right\}$ by permutation. In particular, the sum $\phi_{1}+\cdots+\phi_{s}$ is $G$-invariant. By Claim 2.4, this implies that

$$
\mathcal{P}_{G ; H ; Q}^{!}(\phi)=\left(\phi_{1}+\cdots+\phi_{s}\right) / s \in \mathcal{P X}_{G}(H) .
$$

4) If $\phi \in \mathcal{P X}_{G}(H)$, then for every $g \in G$ we have $g^{!}(\phi)=\phi$ by Claim 2.4, It follows that $H_{i}^{!}(\phi)=\phi$ and

$$
\mathcal{P}_{G ; H ; Q}^{!}(\phi)=\frac{H_{\mathrm{i}}^{!}(\phi)+\cdots+H_{s}^{!}(\phi)}{s}=\frac{s \cdot \phi}{s}=\phi .
$$

Thus, Claim 2.5 is proved.

2.6. Claim. Let $\Lambda_{s}^{*}$ be the operator from the space of functions on $G$ to the space of functions on $H$ induced by the mapping $\Lambda_{s}$. Then $\Lambda_{s}^{*}$ sends isomorphically $\mathcal{P} \mathcal{X}_{G}(H)$ onto $\mathcal{P X}(G)$.

Proof of Claim 2.6. Since $g^{s} \in H$ for every $g \in G$, the definition of a pseudocharacter implies by condition (2) that every pseudocharacter $\phi: G \rightarrow \mathbb{R}$ is uniquely determined by its restriction $\left.\phi\right|_{H}: H \rightarrow \mathbb{R}$. This means that the operator $\mathcal{J}^{!}: \mathcal{P X}(G) \rightarrow \mathcal{P X}(H)$ induced by the natural inclusion $\mathcal{J}: H \rightarrow G$ is injective and sends isomorphically $\mathcal{P X}(G)$ onto $\mathcal{P X}_{G}(H)$.

Moreover, the definition of pseudocharacters implies by condition (2) that the composition $\mathcal{J} \circ \Lambda_{s}: G \rightarrow \mathbb{R} G\left(g \mapsto g^{s} / s\right)$ is pseudohomomorphic, and the corresponding ring operator $\left(\mathcal{J} \circ \Lambda_{s}\right)$ ! is the identity operator on $\mathcal{P X}(G)$.

Since $\left(\mathcal{J} \circ \Lambda_{s}\right)^{!}=\Lambda_{s}^{*} \circ \mathcal{J}^{!}$by definition, it follows that $\Lambda_{s}^{*}$ sends isomorphically $\mathcal{P} \mathcal{X}_{G}(H)$ onto $\mathcal{P X}(G)$. Claim 2.6 is proved.

Now we deduce Proposition 2.1 from the above claims. (Recall that for a map $f$ from a group $G_{1}$ to the group ring $\mathbb{R} G_{2}$ of a group $G_{2}$ we denote by $f^{*}$ the induced operator from the space of functions on $G_{2}$ to the space of functions on $G_{1}$. The map $f$ is said to be pseudohomomorphic if $f^{*}\left(\mathcal{P X}\left(G_{2}\right)\right) \subset \mathcal{P X}\left(G_{1}\right)$.) The identity $\mathcal{T}_{G ; H ; Q}=\mathcal{P}_{G ; H ; Q} \circ \Lambda_{s}$ implies that $\mathcal{T}_{G ; H ; Q}^{*}=\Lambda_{s}^{*} \circ \mathcal{P}_{G ; H ; Q}^{*}$. By assertions 1, 3, and 4 of Claim 2.5, we have

$$
\mathcal{P}_{G ; H ; Q}^{*}(\mathcal{P X}(H))=\mathcal{P}_{G ; H ; Q}^{!}(\mathcal{P X}(H))=\mathcal{P X}_{G}(H) .
$$

Then, by Claim 2.6.

$$
\mathcal{T}_{G ; H ; Q}^{*}(\mathcal{P X}(H))=\Lambda_{s}^{*}\left(\mathcal{P}_{G ; H ; Q}^{*}(\mathcal{P X}(H))\right)=\Lambda_{s}^{*}\left(\mathcal{P} \mathcal{X}_{G}(H)\right)=\mathcal{P X}(G) .
$$

This shows that the mapping $\mathcal{T}_{G ; H ; Q}$ is pseudohomomorphic. The ring operator $\mathcal{T}_{G ; H ; Q}^{!}$: $\mathcal{P X}(H) \rightarrow \mathcal{P X}(G)$ does not depend on $Q$, because by the above we have $\mathcal{T}_{G ; H ; Q}^{!}=$ $\Lambda_{s}^{*} \circ \mathcal{P}_{G ; H ; Q}^{!}$, while neither $\Lambda_{s}^{*}$ nor $\mathcal{P}_{G ; H ; Q}^{!}$depends on $Q$ (see assertion 2 of Claim [2.5). This proves Proposition 2.1.

\section{§3. A RING OPERATOR FOR BRAID GROUPS}

The transfer of pseudocharacters allows us to construct nontrivial ring operators in spaces of pseudocharacters of braid groups. Recall that the pure braid group $P_{n} \subset$ $B_{n}$ is a normal subgroup of index $n$ ! in the braid group $B_{n}$. Therefore, we have the transfer operator $\mathcal{T}_{B_{n} ; P_{n}}^{!}: \mathcal{P X}\left(P_{n}\right) \rightarrow \mathcal{P X}\left(B_{n}\right)$. At the same time, there are various homomorphisms from $P_{n}$ to $B_{n}$ and to braid groups of other ranks that induce nontrivial ring operators on the spaces of pseudocharacters. Combining these ring operators with the transfer $\mathcal{T}_{B_{n} ; P_{n}}^{!}$, we obtain pseudohomomorphic mappings for the pseudocharacters of braid groups. In this section, we apply the described pattern to construct a ring operator 
$\widetilde{\mathcal{R}}_{n, k}^{!}: \mathcal{P X}\left(B_{k}\right) \rightarrow \mathcal{P X}\left(B_{n}\right)$ for spaces of pseudocharacters of braid groups $B_{k}$ and $B_{n}$ with $k<n$. It will be shown in $\S 9$ that operator $\widetilde{\mathcal{R}}_{n, k}^{!}$is injective.

3.1. Pure braids. Let $\Sigma_{\infty}$ be the group of finite permutations of $\mathbb{N}$, and let $\Sigma_{n} \subset \Sigma_{\infty}$ be the group of permutations of the set $\{1, \ldots, n\}$. It is well known that the map $\left\{\sigma_{i}\right\}_{i \in \mathbb{N}} \rightarrow \Sigma_{\infty}$ sending $\sigma_{i}$ to the permutation $(i, i+1)$ extends to an epimorphism $\Sigma: B_{\infty} \rightarrow \Sigma_{\infty}$ such that $\Sigma\left(B_{n}\right)=\Sigma_{n}$. The kernel of $\Sigma$ is the pure braid group of infinite rank $P_{\infty}$. The braids in $P_{\infty}$ are said to be pure. The subgroup $P_{\infty} \cap B_{n}(n \geq 0)$ is the pure braid group of rank $n$. The group $P_{n}$ is a normal subgroup of index $\left|\Sigma_{n}\right|=n$ ! in $B_{n}$. It follows that the braid $\beta^{n !}$ is pure for each $\beta \in B_{n}$.

3.2. Definition: the homomorphism $\mathcal{K}_{n, k}: P_{n} \rightarrow P_{k}$. We introduce the following notation: for $i$ and $j$ with $1 \leq i<j$, we set

$$
a_{i j}:=\sigma_{i} \sigma_{i+1} \cdots \sigma_{j-2} \sigma_{j-1}^{2} \sigma_{j-2}^{-1} \cdots \sigma_{i+1}^{-1} \sigma_{i}^{-1} \in B_{\infty} .
$$

It is known (see, e.g., [12]) that the set $\left\{a_{i j}: 1 \leq i<j \leq m\right\}$ generates $P_{m}$. The elements $a_{i j}$ will be called the Markov generators.

For $0 \leq k<n$, we denote by $H_{n, k}$ the subgroup of $B_{\infty}$ generated by the elements

$$
\left\{a_{i j}: 1 \leq i<j \leq n, k+1 \leq j \leq n\right\} .
$$

It is known that $H_{n, k}$ is a normal subgroup in $P_{n}$. Moreover, $P_{n}$ is a semidirect product of $H_{n, k}$ and $P_{k}$. Let $\mathcal{K}_{n, k}$ be the homomorphism $P_{n} \rightarrow P_{k}$ with $\left.\mathcal{K}_{n, k}\right|_{P_{k}}=\mathrm{id}_{P_{k}}$ and $\operatorname{ker}\left(\mathcal{K}_{n, k}\right)=H_{n, k}$.

3.3. Definition: the operator $\widetilde{\mathcal{R}}_{n, k}^{!}$. We consider the following mappings.

1. The natural inclusion $\mathcal{J}_{k}: P_{k} \rightarrow B_{k}$ with the induced operator

$$
\mathcal{J}_{k}^{!}: \mathcal{P X}\left(B_{k}\right) \rightarrow \mathcal{P X}\left(P_{k}\right) \text {. }
$$

2. The epimorphism $\mathcal{K}_{n, k}: P_{n} \rightarrow P_{k}$ with the induced operator

$$
\mathcal{K}_{n, k}^{!}: \mathcal{P X}\left(P_{k}\right) \rightarrow \mathcal{P X}\left(P_{n}\right)
$$

3. The transfer operator (see Proposition 2.1)

$$
\mathcal{T}_{B_{n} ; P_{n}}^{!}: \mathcal{P X}\left(P_{n}\right) \rightarrow \mathcal{P X}\left(B_{n}\right) .
$$

We define the operator $\widetilde{\mathcal{R}}_{n, k}^{!}: \mathcal{P X}\left(B_{k}\right) \rightarrow \mathcal{P X}\left(B_{n}\right)$ as the composition of the operators $\mathcal{J}_{k}^{!}, \mathcal{K}_{n, k}^{!}$, and $\mathcal{T}_{B_{n} ; P_{n}}^{!}$:

$$
\widetilde{\mathcal{R}}_{n, k}^{!}:=\mathcal{T}_{B_{n} ; P_{n}}^{!} \circ \mathcal{K}_{n, k}^{!} \circ \mathcal{J}_{k}^{!}: \mathcal{P X}\left(B_{k}\right) \rightarrow \mathcal{P X}\left(B_{n}\right) .
$$

We have

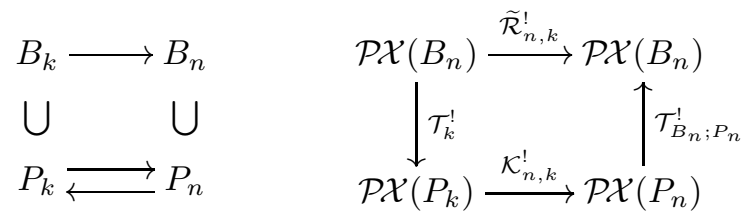

It is clear that $\widetilde{\mathcal{R}}_{n, k}^{!}$is a ring operator (because it is a composition of ring operators). 


\section{§4. Strand REMOVIng PROCEDURES}

In this section, we introduce an auxiliary family of maps red $J: B_{\infty} \rightarrow B_{\infty}(J \subset \mathbb{N})$. These maps are called strand removing procedures. In subsequent sections, we use these procedures to construct pseudohomomorphic maps for braid groups. In particular, we shall describe the ring operator $\widetilde{\mathcal{R}}_{n, k}^{!}$in terms of these procedures. The basic properties of the maps red $J$ allow us to prove various properties of the corresponding pseudohomomorphic mappings and induced ring operators. We define strand removing procedures in terms of the classical geometric interpretation of braids.

4.1. Geometric braids. A geometric braid on $n$ strands is a set of $n$ disjoint curves $\gamma_{i}:[0,1] \rightarrow \mathbb{R}^{3}, i \in\{1, \ldots, n\}$, such that for each curve $\gamma_{i}=:\left(\gamma_{i}^{x}, \gamma_{i}^{y}, \gamma_{i}^{z}\right)$ we have $\gamma_{i}^{x}(t)=t, \gamma_{i}^{z}(0)=\gamma_{i}^{z}(1)=0$. A geometric braid $\left\{\gamma_{1}, \ldots, \gamma_{n}\right\}$ is uniquely determined by its image $\gamma_{1}([0,1]) \cup \cdots \cup \gamma_{n}([0,1]) \subset \mathbb{R}^{3}$, and in certain cases we treat it as a subset in $\mathbb{R}^{3}$.

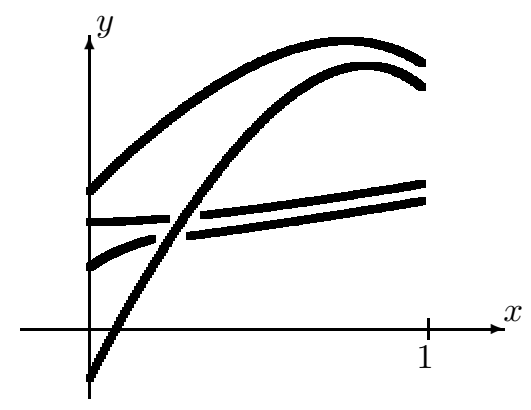

Figure 1. The projection of a geometric braid to the plane $z=0$.

The set of geometric braids is endowed with a natural topology (being a subspace of the topological space of compact subsets in $\mathbb{R}^{3}$ ). The connected components of the topological space of geometric braids are called (isotopic) classes of geometric braids. We denote by $\mathbf{B}_{n}$ the set of classes of geometric braids on $n$ strands.

We do not require a geometric braid $\mathbf{b}=\left\{\gamma_{1}, \ldots, \gamma_{n}\right\}$ to have the sets $\mathbf{b}_{\mid 0}:=$ $\left\{\gamma_{1}^{y}(0), \ldots, \gamma_{n}^{y}(0)\right\}$ and $\mathbf{b}_{\mid 1}:=\left\{\gamma_{1}^{y}(1), \ldots, \gamma_{n}^{y}(1)\right\}$ equal to each other, as opposed to the usual geometric interpretation of braids. It is clear, though, that every class of geometric braids contains an element $\mathbf{b}$ with $\mathbf{b}_{\mid 0}=\mathbf{b}_{\mid 1}$; moreover, every class of geometric braids on $n$ strands contains an element $\mathbf{b}$ with $\mathbf{b}_{\mid 0}=\mathbf{b}_{\mid 1}=\{1, \ldots, n\}$. A geometric braid with $\mathbf{b}_{\mid 0}=\mathbf{b}_{\mid 1}=\{1, \ldots, n\}$ will be called a standard braid.

Let $\mathbf{b} \subset \mathbb{R}^{3}$ and $\mathbf{b}^{\prime} \subset \mathbb{R}^{3}$ be two of the standard geometric braids on $n$ strands. Then, obviously, the set $P\left(\mathbf{b}, \mathbf{b}^{\prime}\right):=D_{1 / 2}\left(\mathbf{b} \cup E_{1}\left(\mathbf{b}^{\prime}\right)\right)$, where $D_{1 / 2}$ and $E_{1}$ are homeomorphisms of $\mathbb{R}^{3}$ defined by $(x, y, z) \mapsto(x / 2, y, z)$ and $(x, y, z) \mapsto(x+1, y, z)$, respectively, is a standard geometric braid on $n$ strands.

Given two classes $\beta, \beta^{\prime} \in \mathbf{B}_{n}$, we define their product $\beta \times \beta^{\prime}$ to be the class in $\mathbf{B}_{n}$ containing the geometric braid $P\left(\mathbf{b}, \mathbf{b}^{\prime}\right)$, where $\mathbf{b}$ and $\mathbf{b}^{\prime}$ are arbitrary standard braids from $\beta$ and $\beta^{\prime}$, respectively. It is well known that the product is well defined, and the set $\mathbf{B}_{n}$ with the operation $\times$ is a group isomorphic to the braid group $B_{n}$. It is also known that the map $\left\{\sigma_{1}, \ldots, \sigma_{n-1}\right\} \rightarrow \mathbf{B}_{n}$ sending $\sigma_{i}$ to the class containing the geometric braid given in Figure 1 extends to a group isomorphism $B_{n} \rightarrow \mathbf{B}_{n}$. We fix this isomorphism and call it the canonical one. 


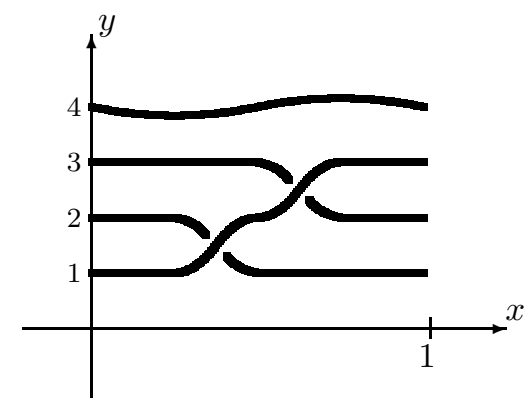

FIGURE 2. The projection of a standard braid.
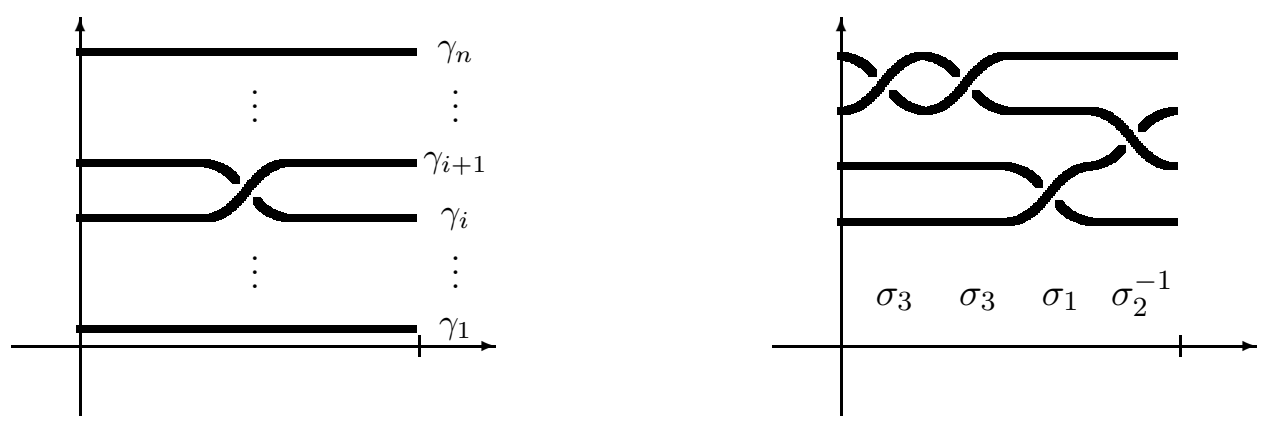

FiguRE 3. Projections of geometric braids representing the braids $\sigma_{i} \in B_{n}$ and $\sigma_{3} \sigma_{3} \sigma_{1} \sigma_{2}^{-1} \in B_{4}$.

Definition: strand removing procedures. Let $J \subset \mathbb{N}$. For a geometric braid $\mathbf{b}=$ $\left\{\gamma_{1}, \ldots, \gamma_{n}\right\}$ on $n$ strands numbered so that $\gamma_{i}^{y}(1)<\gamma_{j}^{y}(1)$ if $i<j$, we denote by $\operatorname{red}_{J}(\mathbf{b})$ the geometric braid $\left\{\gamma_{k}: k \in\{1, \ldots, n\} \backslash J\right\}$ on $n-|J \cap\{1, \ldots, n\}|$ strands.

Clearly, if a geometric braid $\mathbf{b}$ is in the class of a geometric braid $\mathbf{b}^{\prime}$, then $\operatorname{red}_{J}(\mathbf{b})$ is in the class of $\operatorname{red}_{J}\left(\mathbf{b}^{\prime}\right)$. Moreover, a standard argument shows that if a geometric braid $\mathbf{b}$ on $m$ strands and a geometric braid $\mathbf{b}^{\prime}$ on $m^{\prime}$ strands represent 11 one and the same braid of $B_{\infty}$, then $\operatorname{red}_{J}(\mathbf{b})$ and $\operatorname{red}_{J}\left(\mathbf{b}^{\prime}\right)$ also represent one and the same braid of $B_{\infty}$. This shows that we can define strand removing procedures for braids of $B_{\infty}$.

For $J \subset \mathbb{N}$, we define red $J: B_{\infty} \rightarrow B_{\infty}$ as the mapping that sends a braid $\beta \in B_{\infty}$ to the braid represented by the geometric braid $\operatorname{red}_{J}(\mathbf{b})$, where $\mathbf{b}$ is an arbitrary geometric braid representing $\beta$.

\section{§5. Properties of Strand REMoving Procedures AND THE DEFinition OF THE HOMOMORPHISMS RED}

We introduce auxiliary notation.

5.1. Notation: the symmetric group. For a braid $\beta \in B_{\infty}$ and a subset $J \subset \mathbb{N}$, we denote by $\beta(J)$ the image of $J$ under the permutation $\Sigma(\beta)$, where $\Sigma: B_{\infty} \rightarrow \Sigma_{\infty}$ is the epimorphism described in Subsection 3.1 .

\footnotetext{
${ }^{1}$ Every geometric braid $\mathbf{d}$ on $k$ strands $(k \in \mathbb{N})$ represents a certain braid in $B_{\infty}$, because $\mathbf{d}$ represents a braid in $B_{k}$, while $B_{k} \subset B_{\infty}$.
} 


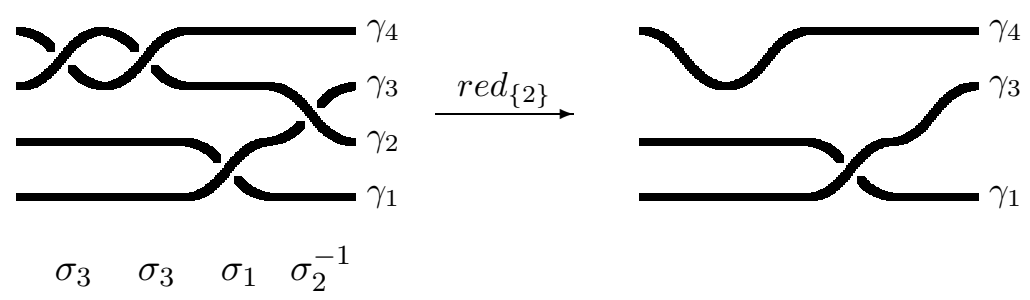

FiguRE 4. Removing a strand.

5.2. Notation: removal of subsets. Let $I \subset \mathbb{N}$. Let $R_{I}$ denote the map $\mathbb{N} \backslash I \rightarrow \mathbb{N}$ that sends $m \in \mathbb{N} \backslash I$ to

$$
R_{I}(m):=m-|\{1, \ldots, m\} \cap I| .
$$

For subsets $I$ and $J$ of $\mathbb{N}$, we put

$$
J / I:=R_{I}(J \backslash I) .
$$

(In particular, we have $\{m\} / I=\varnothing$ if $m \in I$.)

5.3. Convention. To shorten the notation, from now on we drop curly brackets in descriptions of sets (in cases where this does not cause any confusion); e.g., we write $m / I$ instead of $\{m\} / I$ and $\beta(2,3)$ instead of $\beta(\{2,3\})$.

The following lemmas can easily be deduced from the definitions.

5.4. Lemma. Let $J \subset \mathbb{N}, i \in \mathbb{N}, \delta \in\{+1,-1\}$. Then

$$
\operatorname{red}_{J}\left(\sigma_{i}^{\delta}\right)= \begin{cases}1 & \text { if either } i \in J \text { or } i+1 \in J, \\ \sigma_{i / J}^{\delta} & \text { if }\{i, i+1\} \cap J=\varnothing .\end{cases}
$$

5.5. Lemma. Let $J \subset \mathbb{N}, i<j \in \mathbb{N}$, and let $a_{i j}$ be a Markov generator (see Definition 3.2). Then

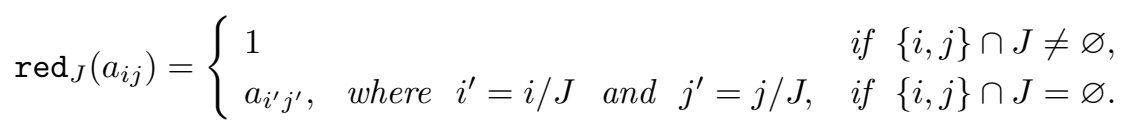

5.6. Lemma. Let $J \subset\{1, \ldots, n\}$. Then

$$
\operatorname{red}_{J}\left(B_{n}\right)=B_{n-|J|} .
$$

5.7. Lemma. Let $J \subset\{n+1, n+2, \ldots\}, \beta \in B_{n}$. Then

$$
\operatorname{red}_{J}(\beta)=\beta \text {. }
$$

5.8. Lemma. Let $J \subset \mathbb{N}, \alpha, \beta \in B_{\infty}$. Then

$$
\operatorname{red}_{J}(\alpha \beta)=\operatorname{red}_{\beta(J)}(\alpha) \cdot \operatorname{red}_{J}(\beta) .
$$

5.9. Lemma. Let $I, J \subset \mathbb{N}$. Then

$$
\operatorname{red}_{I \cup J} \equiv \operatorname{red}_{I / J} \circ \operatorname{red}_{J} .
$$

5.10. Remark. The family of mappings red is uniquely determined by the properties described in Lemmas 5.4 and 5.8. In particular, these two lemmas imply all the other lemmas of this section.

5.11. Remark. Lemmas 5.4 and 5.8 provide us with an algorithm for computing $\operatorname{red}_{J}(\beta)$ (given a word over the Artin generators and their inverses that represents $\beta$ ). 
5.12. Definition: the homomorphisms RED. We denote by $\mathrm{RED}_{J}$ the restriction of red $J$ to the subgroup $P_{\infty} \subset B_{\infty}$ of pure braids.

Obviously, Lemmas 5.5 and 5.8 imply that for every $J \subset \mathbb{N}$ the mapping

$$
\mathrm{RED}_{J}: P_{\infty} \rightarrow B_{\infty}
$$

is a homomorphism. Furthermore, the image of the homomorphism lies in $P_{\infty}$.

5.13. Corollary. Let $0 \leq k<n$. Then the restriction $\left.\operatorname{RED}_{\{k+1, \ldots, n\}}\right|_{P_{n}}$ coincides with the homomorphism $\mathcal{K}_{n, k}: P_{n} \rightarrow P_{k}$ (see Definition 3.2).

Proof of Corollary 5.13. It suffices to show that the restrictions of the homomorphisms $\mathcal{K}_{n, k}$ and $\operatorname{RED}_{\{k+1, \ldots, n\}}$ to the set $\left\{a_{i j}: 1 \leq i<j \leq n\right\}$ of the Markov generators coincide. (It was mentioned in Subsection 3.2 that this set generates $P_{n}$.) By Definition 3.2 and Lemma 5.5. each of the mappings $\mathcal{K}_{n, k}$ and $\operatorname{RED}_{\{k+1, \ldots, n\}}$ induces the identity mapping on the set $\left\{a_{i j}: 1 \leq i<j \leq k\right\}$ and sends the set $\left\{a_{i j}: 1 \leq i<j \leq n\right.$, $k+1 \leq j \leq n\}$ (which generates the subgroup $H_{n, k}$ ) to the group's identity. Therefore, $\left.\operatorname{RED}_{\{k+1, \ldots, n\}}\right|_{P_{n}}=\mathcal{K}_{n, k}$.

\section{§6. The OPERATORS $\mathcal{R}_{n, k}^{!}$}

In this section, we introduce a pseudohomomorphic mapping $\mathcal{R}_{n, k}: B_{n} \rightarrow \mathbb{R} B_{k}$ $(k<n)$ (which is defined in terms of strand removing procedures) and show that the corresponding ring operator $\mathcal{R}_{n, k}^{!}: \mathcal{P X}\left(B_{k}\right) \rightarrow \mathcal{P X}\left(B_{n}\right)$ coincides with the operator $\left(\begin{array}{l}n \\ k\end{array}\right) \cdot \widetilde{\mathcal{R}}_{n, k}^{!}$, where $\widetilde{\mathcal{R}}_{n, k}^{!}$is the operator defined in $\S 3$ and $\left(\begin{array}{l}n \\ k\end{array}\right)=\frac{n !}{k !(n-k) !}$.

6.1. Definition: the mapping $\mathcal{R}_{n, k}$. Let $0 \leq k<n$. We define a mapping $\mathcal{R}_{n, k}$ : $B_{n} \rightarrow \mathbb{R} B_{k}$ as follows:

$$
\begin{aligned}
& \mathcal{R}_{n, k}(\beta):=\sum_{J \in \mathbf{C}_{n}^{n-k}} \frac{\operatorname{RED}_{J}\left(\beta^{n !}\right)}{n !}, \\
& \text { where } \quad \mathbf{C}_{n}^{n-k}:=\{I \subset\{1, \ldots, n\}:|I|=n-k\} .
\end{aligned}
$$

6.2. Proposition. The mapping $\mathcal{R}_{n, k}$ is pseudohomomorphic. The induced ring operator $\mathcal{R}_{n, k}^{!}: \mathcal{P X}\left(B_{k}\right) \rightarrow \mathcal{P X}\left(B_{n}\right)$ coincides with the operator $\left(\begin{array}{l}n \\ k\end{array}\right) \cdot \widetilde{\mathcal{R}}_{n, k}^{!}$.

We need the following lemma to prove Proposition 6.2 .

6.3. Lemma. Assume that two pure braids $\gamma_{1}$ and $\gamma_{2}$ in $P_{n} \subset B_{n}$ are conjugate in $B_{n}$ by a braid $\alpha$ :

$$
\gamma_{2}=\alpha \gamma_{1} \alpha^{-1}
$$

Then for every $J \subset\{1, \ldots, n\}$ the braids $\operatorname{RED}_{J}\left(\gamma_{1}\right)$ and $\operatorname{RED}_{\alpha(J)}\left(\gamma_{2}\right)$ (by Lemma 5.6 and the remark after Definition 5.12 , these braids lie in the subgroup $\left.B_{n-|J|} \cap P_{\infty}=P_{n-|J|}\right)$ are conjugate by a braid in $B_{n-|J|}$.

Proof of Lemma 6.3. By Lemma 5.8, the identity $\gamma_{2}=\alpha \gamma_{1} \alpha^{-1}$ implies that

$$
\begin{aligned}
\operatorname{RED}_{\alpha(J)}\left(\gamma_{2}\right) & =\operatorname{RED}_{\alpha(J)}\left(\alpha \gamma_{1} \alpha^{-1}\right) \\
& =\operatorname{red}_{\gamma_{1} \alpha^{-1} \alpha(J)}(\alpha) \cdot \operatorname{RED}_{\alpha^{-1} \alpha(J)}\left(\gamma_{1}\right) \cdot \operatorname{red}_{\alpha(J)}\left(\alpha^{-1}\right) .
\end{aligned}
$$

Since the permutations $\Sigma\left(\alpha^{-1} \alpha\right)$ and $\Sigma\left(\gamma_{1} \alpha^{-1} \alpha\right)$ are trivial (because the braid $\gamma_{1}$ is pure), we can rewrite (3) in the form

$$
\operatorname{RED}_{\alpha(J)}\left(\gamma_{2}\right)=\operatorname{red}_{J}(\alpha) \cdot \operatorname{RED}_{J}\left(\gamma_{1}\right) \cdot \operatorname{red}_{\alpha(J)}\left(\alpha^{-1}\right) .
$$

By Lemma 5.8 , the identity $1=\alpha^{-1} \alpha$ implies that

$$
1=\operatorname{red}_{J}(1)=\operatorname{red}_{J}\left(\alpha^{-1} \alpha\right)=\operatorname{red}_{\alpha(J)}\left(\alpha^{-1}\right) \cdot \operatorname{red}_{J}(\alpha) .
$$


Therefore, $\operatorname{red}_{\alpha(J)}\left(\alpha^{-1}\right)=\left(\operatorname{red}_{J}(\alpha)\right)^{-1}$. This allows us to rewrite (4) in the form

$$
\operatorname{RED}_{\alpha(J)}\left(\gamma_{2}\right)=\operatorname{red}_{J}(\alpha) \cdot \operatorname{RED}_{J}\left(\gamma_{1}\right) \cdot\left(\operatorname{red}_{J}(\alpha)\right)^{-1} .
$$

By Lemma 5.6, the braid $\operatorname{red}_{J}(\alpha)$ is in the subgroup $B_{n-|J|}$. This completes the proof.

Proof of Proposition 6.2. Let $Q \subset B_{n}$ be a set of representatives of the cosets of $P_{n}$ in $B_{n}$, i.e., a set that is mapped injectively by the homomorphism $\Sigma: B_{n} \rightarrow \Sigma_{n}$ onto the symmetric group $\Sigma_{n}$. Then the definition of $\widetilde{\mathcal{R}}_{n, k}^{!}$implies that this operator is induced by the following pseudohomomorphic mapping $\widetilde{\mathcal{R}}_{Q}$ :

$$
\widetilde{\mathcal{R}}_{Q}: B_{n} \rightarrow \mathbb{R} B_{k}, \quad \widetilde{\mathcal{R}}_{Q}(\beta):=\sum_{q \in Q} \frac{\mathcal{K}_{n, k}\left(q \beta^{n !} q^{-1}\right)}{(n !)^{2}} .
$$

By Corollary [5.13. $\mathcal{K}_{n, k}=\left.\operatorname{RED}_{\{k+1, \ldots, n\}}\right|_{P_{n}}$. Hence, for every $\beta \in B_{n}$, we have

$$
\widetilde{\mathcal{R}}_{Q}(\beta)=\sum_{q \in Q} \frac{\mathcal{K}_{n, k}\left(q \beta^{n !} q^{-1}\right)}{(n !)^{2}}=\sum_{q \in Q} \frac{\operatorname{RED}_{\{k+1, \ldots, n\}}\left(q \beta^{n !} q^{-1}\right)}{(n !)^{2}} .
$$

Using Lemma 6.3, we see that the braid $\operatorname{RED}_{\{k+1, \ldots, n\}}\left(q \beta^{n !} q^{-1}\right)$ is conjugate in $B_{k}$ to the braid $\operatorname{RED}_{q^{-1}(k+1, \ldots, n)}\left(\beta^{n !}\right)$. Since every pseudocharacter is conjugacy invariant, it follows that the mapping

$$
\widetilde{\mathcal{R}}_{Q}^{\prime}: B_{n} \rightarrow \mathbb{R} B_{k}, \quad \widetilde{\mathcal{R}}_{Q}^{\prime}(\beta):=\sum_{q \in Q} \frac{\operatorname{RED}_{q^{-1}(k+1, \ldots, n)}\left(\beta^{n !}\right)}{(n !)^{2}}
$$

is also pseudohomomorphic and is equivalent to the mapping $\widetilde{\mathcal{R}}_{Q}$ (i.e., $\widetilde{\mathcal{R}}_{Q}^{\prime !}=\widetilde{\mathcal{R}}_{Q}^{!}=$ $\widetilde{\mathcal{R}}_{n, k}^{!}$).

Next, we observe that

$$
\sum_{q \in Q} \frac{\operatorname{RED}_{q^{-1}(k+1, \ldots, n)}\left(\beta^{n !}\right)}{(n !)^{2}}=\sum_{s \in \Sigma_{n}} \frac{\operatorname{RED}_{s(k+1, \ldots, n)}\left(\beta^{n !}\right)}{(n !)^{2}}
$$

and

$$
\sum_{s \in \Sigma_{n}} \frac{\operatorname{RED}_{s(k+1, \ldots, n)}\left(\beta^{n !}\right)}{(n !)^{2}}=\sum_{J \in \mathbf{C}_{n}^{n-k}}\left((n-k) ! \cdot k ! \cdot \frac{\operatorname{RED}_{J}\left(\beta^{n !}\right)}{(n !)^{2}}\right)=\frac{\mathcal{R}_{n, k}(\beta)}{\left(\begin{array}{l}
n \\
k
\end{array}\right)} .
$$

Therefore, $\widetilde{\mathcal{R}}_{Q}^{\prime}=\mathcal{R}_{n, k} /\left(\begin{array}{l}n \\ k\end{array}\right)$, i.e., the mapping $\mathcal{R}_{n, k}$, is pseudohomomorphic, likewise $\widetilde{\mathcal{R}}_{Q}^{\prime}$, and

$$
\mathcal{R}_{n, k}^{!}=\left(\begin{array}{l}
n \\
k
\end{array}\right) \cdot \widetilde{\mathcal{R}}_{Q}^{\prime !}=\left(\begin{array}{l}
n \\
k
\end{array}\right) \cdot \widetilde{\mathcal{R}}_{Q}^{!}=\left(\begin{array}{l}
n \\
k
\end{array}\right) \cdot \widetilde{\mathcal{R}}_{n, k}^{!} .
$$

6.4. Remark. Given a computational algorithm for a pseudocharacter $\phi: B_{k} \rightarrow \mathbb{R}$, we can easily construct an algorithm for the pseudocharacter $\mathcal{R}_{n, k}^{!}(\phi)$. To simplify the latter algorithm by avoiding computation of $\phi\left(\operatorname{RED}_{J}\left(\beta^{n !}\right)\right)$ with a "large" braid $\beta^{n !}$, we can use the following observations.

If $\beta \in B_{n}, s \neq 0$, and the braid $\beta^{s}$ is pure, then for each $J \subset \mathbf{C}_{n}^{n-k}$ we have

$$
\phi\left(\operatorname{RED}_{J}\left(\beta^{n !}\right)\right) / n !=\phi\left(\operatorname{RED}_{J}\left(\beta^{s}\right)\right) / s .
$$

Furthermore, if $\beta \in B_{n}, J \subset \mathbf{C}_{n}^{n-k}, s_{J} \neq 0$, and $\beta^{s_{J}}(J)=J$, then

$$
\phi\left(\operatorname{RED}_{J}\left(\beta^{n !}\right)\right) / n !=\phi\left(\operatorname{RED}_{J}\left(\beta^{s_{J}}\right)\right) / s_{J} .
$$


6.5. Remark. Let $0 \leq k<n$, and let $\mathcal{P}_{n, k}: B_{n} \rightarrow \mathbb{R} B_{k}$ be the mapping defined as follows:

$$
\mathcal{P}_{n, k}(\beta):=\sum_{J \in \mathbf{C}_{n}^{n-k}} \operatorname{RED}_{J}(\beta) .
$$

The mapping $\mathcal{P}_{n, k}$ is not pseudohomomorphic in general. However, it is quasihomomorphic: for every quasimorphism $\psi: B_{k} \rightarrow \mathbb{R}$ the function $\mathcal{P}_{n, k}^{*}(\psi): B_{n} \rightarrow \mathbb{R}$ (this function sends $\beta \in B_{n}$ to $\left.\sum_{J \in \mathbf{C}_{n}^{n-k}} \psi\left(\operatorname{RED}_{J}(\beta)\right)\right)$ is a quasimorphism. Observe that the difference $\mathcal{P}_{n, k}^{*}(\phi)-\mathcal{R}_{n, k}^{!}(\phi)$ is bounded for every pseudocharacter $\phi: B_{k} \rightarrow \mathbb{R}$.

\section{§. The operators $\mathcal{A}_{n, k}^{!}$And the surjeCtivity of $\mathcal{I}_{n, k}^{!}$}

7.1. Definition: the operators $\mathcal{A}_{n, k}^{!}$. For $m>0$, we define a mapping $\mathcal{A}_{m, m-1}$ from the group $B_{m}$ to the group ring $\mathbb{R} B_{m-1}$ as follows:

$$
\mathcal{A}_{m, m-1}:=\sum_{k=0,1, \ldots, m-1}(-1)^{m-k+1} \cdot \mathcal{R}_{m, k},
$$

where $\mathcal{R}_{m, k}$ is the mapping $B_{m} \rightarrow \mathbb{R} B_{k}$ defined in Subsection 6.1 (here, we use the fact that $B_{k} \subset B_{m-1}$, and consequently, $\mathbb{R} B_{k} \subset \mathbb{R} B_{m-1}$ for $\left.k=0,1, \ldots, m-1\right)$. Note that by the definition of $\mathcal{R}_{m, k}$ we have

$$
\mathcal{A}_{m, m-1}(\beta)=\sum_{\{J \subset\{1, \ldots, m\}: J \neq \varnothing\}} \frac{(-1)^{|J|+1} \cdot \operatorname{RED}_{J}\left(\beta^{m !}\right)}{m !} .
$$

The mapping $\mathcal{A}_{m, m-1}$ is pseudohomomorphic because it is a linear combination of pseudohomomorphic mappings. We consider the induced ring operator

$$
\mathcal{A}_{m, m-1}^{!}: \mathcal{P X}\left(B_{m-1}\right) \rightarrow \mathcal{P X}\left(B_{m}\right) .
$$

Formula (7) shows that

$$
\mathcal{A}_{m, m-1}^{!}=\mathcal{R}_{m, m-1}^{!}+\sum_{0 \leq k \leq m-2}(-1)^{m-k+1} \cdot \mathcal{R}_{m, k}^{!} \circ \mathcal{I}_{m-1, k}^{!} .
$$

For $0 \leq k<n$, we define the operator $\mathcal{A}_{n, k}^{!}$from $\mathcal{P X}\left(B_{k}\right)$ to $\mathcal{P X}\left(B_{n}\right)$ as follows:

$$
\mathcal{A}_{n, k}^{!}:=\mathcal{A}_{n, n-1}^{!} \circ \mathcal{A}_{n-1, n-2}^{!} \circ \cdots \circ \mathcal{A}_{k+2, k+1}^{!} \circ \mathcal{A}_{k+1, k}^{!} .
$$

7.2. Proposition. Let $0 \leq k<n$. Then $\mathcal{I}_{n, k}^{!} \circ \mathcal{A}_{n, k}^{!}=\operatorname{id}_{\mathcal{P X}\left(B_{k}\right)}$. Therefore, the operator $\mathcal{A}_{n, k}^{!}$is injective, and the operator $\mathcal{I}_{n, k}^{!}$is surjective.

Our proof of Proposition 7.2 is based on the following lemma.

7.3. Lemma (on an alternating sum). Suppose $n>0$ and $\beta \in B_{n-1}$. Then

$$
\sum_{J \subset\{1, \ldots, n\}}(-1)^{|J|} \cdot \operatorname{red}_{J}(\beta)=0 .
$$

(The sum is in the group ring $\mathbb{R} B_{n-1}$.)

Proof of Lemma 7.3. It is easily seen that $I /\{n\}=I$ if $I \subset\{1, \ldots, n-1\}$. (We put $\{1, \ldots, n-1\}:=\varnothing$ for $n=1$.) Then, by Lemmas 5.9 and 5.7

$$
\operatorname{red}_{I \cup\{n\}}(\beta)=\operatorname{red}_{I /\{n\}} \circ \operatorname{red}_{n}(\beta)=\operatorname{red}_{I}(\beta) .
$$

Next, observe that

$$
\{J \mid J \subset\{1, \ldots, n\}\}=\bigcup_{I \subset\{1, \ldots, n-1\}}\{I, I \cup\{n\}\} .
$$


This implies the identities

$$
\sum_{J \subset\{1, \ldots, n\}}(-1)^{|J|} \cdot \operatorname{red}_{J}(\beta) \stackrel{[12 \mid}{=} \sum_{I \subset\{1, \ldots, n-1\}}(-1)^{|I|} \cdot\left(\operatorname{red}_{I}(\beta)-\operatorname{red}_{I \cup\{n\}}(\beta)\right) \stackrel{\sqrt{111}=}{=} 0 .
$$

Proof of Proposition 17.2. The above definition of the operator $\mathcal{A}_{n, k}^{!}$(see (10) ) and the obvious relation

$$
\mathcal{I}_{n, k}^{!}=\mathcal{I}_{k+1, k}^{!} \circ \mathcal{I}_{k+2, k+1}^{!} \circ \cdots \circ \mathcal{I}_{n-1, n-2}^{!} \circ \mathcal{I}_{n, n-1}^{!}
$$

show that to prove the relation $\mathcal{I}_{n, k}^{!} \circ \mathcal{A}_{n, k}^{!}=\operatorname{id}_{\mathcal{P X}\left(B_{k}\right)}$ it suffices to check that $\mathcal{I}_{\ell, \ell-1}^{!} \circ$ $\mathcal{A}_{\ell, \ell-1}^{!}=\operatorname{id}_{\mathcal{P X}\left(B_{\ell-1}\right)}$ for each $\ell \in\{k+1, \ldots, n\}$. In other words, it suffices to prove Proposition 7.2 only in the case where $k=n-1$.

To prove the identity $\mathcal{I}_{\ell, \ell-1}^{!} \circ \mathcal{A}_{\ell, \ell-1}^{!}=\operatorname{id}_{\mathcal{P X}\left(B_{\ell-1}\right)}$, we need to check that $\left(\mathcal{I}_{\ell, \ell-1}^{!} \circ\right.$ $\left.\mathcal{A}_{\ell, \ell-1}^{!}(\phi)\right)(\alpha)=\phi(\alpha)$ for any $\alpha \in B_{\ell-1}, \phi \in \mathcal{P X}\left(B_{\ell-1}\right)$. We shall show that the difference $x:=\phi(\alpha)-\left(\mathcal{I}_{\ell, \ell-1}^{!} \circ \mathcal{A}_{\ell, \ell-1}^{!}(\phi)\right)(\alpha)$ equals zero.

Since $\mathcal{I}_{\ell, \ell-1}$ is an identical inclusion, it follows that

$$
x=\phi(\alpha)-\phi\left(\mathcal{A}_{\ell, \ell-1} \circ \mathcal{I}_{\ell, \ell-1}(\alpha)\right)=\phi(\alpha)-\phi\left(\mathcal{A}_{\ell, \ell-1}(\alpha)\right) .
$$

The definition of a pseudocharacter implies that $\phi(\alpha)=\phi\left(\alpha^{\ell !}\right) / \ell$ !. The definition of the homomorphisms RED implies that $\alpha^{\ell !}=\operatorname{RED}_{\varnothing}\left(\alpha^{\ell !}\right)$. Using these identities and (8), we obtain

$$
\begin{aligned}
x & =\phi(\alpha)-\phi\left(\mathcal{A}_{\ell, \ell-1}(\alpha)\right) \\
& =\phi\left(\frac{\operatorname{RED} \varnothing\left(\alpha^{\ell !}\right)}{\ell !}\right)-\phi\left(\sum_{\{J \subset\{1, \ldots, \ell\}: J \neq \varnothing\}} \frac{(-1)^{|J|+1} \cdot \operatorname{RED}_{J}\left(\alpha^{\ell !}\right)}{\ell !}\right) \\
& =\phi\left(\frac{\operatorname{RED} \varnothing\left(\alpha^{\ell !}\right)}{\ell !}+\sum_{\{J \subset\{1, \ldots, \ell\}: J \neq \varnothing\}} \frac{(-1)^{|J|} \cdot \operatorname{RED}_{J}\left(\alpha^{\ell !}\right)}{\ell !}\right) \\
& =\phi\left(\sum_{J \subset\{1, \ldots, \ell\}} \frac{(-1)^{|J|} \cdot \operatorname{RED}_{J}\left(\alpha^{\ell !}\right)}{\ell !}\right) .
\end{aligned}
$$

Since the braid $\alpha^{\ell !}$ lies in the subgroup $B_{\ell-1}$, Lemma 7.3 shows that

$$
\sum_{J \subset\{1, \ldots, \ell\}} \frac{(-1)^{|J|} \cdot \operatorname{RED}_{J}\left(\alpha^{\ell !}\right)}{\ell !}=0 .
$$

Therefore,

$$
x=\phi(0)=0 .
$$

\section{$\S 8$. The Structure of the SPaCe $\mathcal{P} \mathcal{X}\left(B_{n}\right)$}

8.1. Definition: kernel pseudocharacters. Let $m>0$, and let $\mathcal{I}_{m, m-1}^{!}$be the operator from $\mathcal{P X}\left(B_{m}\right)$ to $\mathcal{P X}\left(B_{m-1}\right)$ induced by the natural inclusion $\mathcal{I}_{m, m-1}: B_{m-1} \rightarrow B_{m}$. We call the kernel of $\mathcal{I}_{m, m-1}^{!}$the space of kernel pseudocharacters of $B_{m}$ and denote it by $\Gamma_{m}$. The pseudocharacters from $\Gamma_{m}$ are said to be kernel.

8.2. Remark. The space $\Gamma_{1}=\mathcal{P X}\left(B_{1}\right)$ is trivial, while $\Gamma_{2}=\mathcal{P X}\left(B_{2}\right) \simeq \mathbb{R}$. It is easy to check that the subspace of kernel pseudocharacters $\Gamma_{3}$ has codimension 1 in $\mathcal{P X}\left(B_{3}\right)$, whence $\Gamma_{3}$ is infinite-dimensional (because $\mathcal{P X}\left(B_{3}\right)$ is infinite-dimensional; see Subsection (0.2). It is natural to conjecture that the space $\Gamma_{m}$ is also infinite-dimensional if $m \geq 4$, but the existence of nontrivial kernel pseudocharacters in $\mathcal{P X}\left(B_{m}\right)$ with $m \geq 4$ is nonobvious a priori. The results of [10], where a series of kernel pseudocharacters was described, show that $\Gamma_{m}$ is nontrivial if $m \geq 4$. 
8.3. Definition: subkernel pseudocharacters. Suppose $0<k<m$. The image $\mathcal{A}_{m, k}^{!}\left(\Gamma_{k}\right) \subset \mathcal{P X}\left(B_{m}\right)$, where $\mathcal{A}_{m, k}^{!}: \mathcal{P X}\left(B_{k}\right) \rightarrow \mathcal{P X}\left(B_{m}\right)$ is the operator defined in Subsection 7.1 will be called the space of subkernel pseudocharacters of degree $k$ for the group $B_{m}$ and will be denoted by $\Gamma_{m, k}$. The nontrivial pseudocharacters in $\Gamma_{m, k}$ are called subkernel pseudocharacters of degree $k$.

In certain cases, we refer to the kernel pseudocharacters of $\mathcal{P X}\left(B_{m}\right)$ as subkernel pseudocharacters of degree $m$ and denote the space $\Gamma_{m}$ by $\Gamma_{m, m}$.

8.4. Remark. If $m>1$, then the space $\Gamma_{m, 1}$ is trivial. It is easy to check that $\Gamma_{m, 2}=$ $\operatorname{Hom}\left(B_{m}, \mathbb{R}\right) \simeq \mathbb{R}$.

8.5. Proposition. The space $\mathcal{P X}\left(B_{n}\right)$ of pseudocharacters of the braid group $B_{n}(n>0)$ is the direct sum of the subspace of kernel pseudocharacters and the subspaces of subkernel pseudocharacters:

$$
\mathcal{P X}\left(B_{n}\right)=\Gamma_{n} \oplus \Gamma_{n, n-1} \oplus \cdots \oplus \Gamma_{n, 2} .
$$

Proof. The proof is by induction on $n$. For $n=1$, the claim is obvious, because the space $\mathcal{P X}\left(B_{1}\right)$ is trivial. Now, let $n>1$ and assume that

$$
\mathcal{P X}\left(B_{n-1}\right)=\Gamma_{n-1} \oplus \Gamma_{n-1, n-2} \oplus \cdots \oplus \Gamma_{n-1,2} .
$$

Proposition 7.2 implies that $\mathcal{P X}\left(B_{n}\right)=\Gamma_{n} \oplus \mathcal{A}_{n, n-1}^{!}\left(\mathcal{P X}\left(B_{n-1}\right)\right)$. Since $\mathcal{A}_{n, n-1}^{!}$is injective (see Proposition 7.2), it follows that

$$
\mathcal{P X}\left(B_{n}\right)=\Gamma_{n} \oplus \mathcal{A}_{n, n-1}^{!}\left(\Gamma_{n-1}\right) \oplus \mathcal{A}_{n, n-1}^{!}\left(\Gamma_{n-1, n-2}\right) \oplus \cdots \oplus \mathcal{A}_{n, n-1}^{!}\left(\Gamma_{n-1,2}\right) .
$$

But by Definition 8.3 we have $\mathcal{A}_{n, n-1}^{!}\left(\Gamma_{n-1}\right)=\Gamma_{n, n-1}$, whence

$$
\mathcal{A}_{n, n-1}^{!}\left(\Gamma_{n-1, k}\right)=\mathcal{A}_{n, n-1}^{!}\left(\mathcal{A}_{n-1, k}^{!}\left(\Gamma_{k}\right)\right)=\mathcal{A}_{n, k}^{!}\left(\Gamma_{k}\right)=\Gamma_{n, k} .
$$

8.6. Definition: $\Gamma$-diagonal operators. Let $m \geq 0$. We say that an operator $X$ : $\mathcal{P X}\left(B_{m}\right) \rightarrow \mathcal{P X}\left(B_{m}\right)$ is $\Gamma$-diagonal if for each $t \in\{1, \ldots, m\}$ the restriction of $X$ to the subspace of subkernel pseudocharacters $\Gamma_{m, t}$ is multiplication by a constant $c_{t}$ (we include the case where $\left.c_{t}=0\right)$. Let $0 \leq k<n$. We say that operators $Y: \mathcal{P X}\left(B_{k}\right) \rightarrow \mathcal{P X}\left(B_{n}\right)$ and $Z: \mathcal{P X}\left(B_{n}\right) \rightarrow \mathcal{P X}\left(B_{k}\right)$ are $\Gamma$-diagonal if the compositions $Y \circ \mathcal{I}_{n, k}^{!}$and $\mathcal{A}_{n, k}^{!} \circ Z$ are $\Gamma$-diagonal.

8.7. Remark. Proposition 7.2 shows immediately that the operators $\mathcal{I}_{n, k}^{!}$and $\mathcal{A}_{n, k}^{!}$are $\Gamma$-diagonal (for any $0 \leq k<n$ ) and the series of operators $\left\{\mathcal{I}_{n, k}^{!}\right\}_{0 \leq k<n}$ and $\left\{\mathcal{A}_{n, k}^{!}\right\}_{0 \leq k<n}$ generate (by compositions and linear combinations) the class of $\Gamma$-diagonal operators. In particular, this means that any $\Gamma$-diagonal operator is a ring operator.

8.8. Remark. Clearly, if $n>0$ and $s>0$, then the set of $\Gamma$-diagonal operators from $\mathcal{P X}\left(B_{n}\right)$ to $\mathcal{P X}\left(B_{s}\right)$ is a linear space of dimension $\min \{n, s\}-1$.

8.9. Remark. It turns out that for any $0 \leq k<n$, the operator $\mathcal{R}_{n, k}^{!}$is $\Gamma$-diagonal (see Proposition 9.3). By Proposition 6.2, this implies that the operator $\widetilde{\mathcal{R}}_{n, k}^{!}$is also $\Gamma$-diagonal. Therefore, the operators of the four series $\left\{\mathcal{I}_{n, k}^{!}\right\}_{0 \leq k<n},\left\{\widetilde{\mathcal{R}}_{n, k}^{!}\right\}_{0 \leq k<n}$, $\left\{\mathcal{R}_{n, k}^{!}\right\}_{0 \leq k<n},\left\{\mathcal{A}_{n, k}^{!}\right\}_{0 \leq k<n}$ are $\Gamma$-diagonal. Furthermore, our results show that the class of $\Gamma$-diagonal operators is generated (by applying compositions and linear combinations) by each of the following three pairs of series: $\left\{\mathcal{I}_{n, k}^{!}\right\}_{0 \leq k<n},\left\{\mathcal{A}_{n, k}^{!}\right\}_{0 \leq k<n}$ (see Remark 8.7); $\left\{\mathcal{I}_{n, k}^{!}\right\}_{0 \leq k<n},\left\{\widetilde{\mathcal{R}}_{n, k}^{!}\right\}_{0 \leq k<n} ;\left\{\mathcal{I}_{n, k}^{!}\right\}_{0 \leq k<n},\left\{\widetilde{\mathcal{R}}_{n, k}^{!}\right\}_{0 \leq k<n}$.

8.10. Remark. Let $1<k<n$. Then every $\Gamma$-diagonal operator $X: \mathcal{P X}\left(B_{k}\right) \rightarrow \mathcal{P X}\left(B_{n}\right)$ is uniquely determined by the sequence of coefficients $c_{2}, c_{3}, \ldots, c_{k}$, where $c_{t}:=c_{t}(X)$ 
is the constant such that for every subkernel pseudocharacter $\phi \in \Gamma_{k, t}$ we have $\mathcal{I}_{n, k}^{!} \circ$ $X(\phi)=c_{t} \cdot \phi$. Lemmas 9.5 and 9.1 imply that

$$
c_{t}\left(\mathcal{R}_{n, k}^{!}\right)=\left(\begin{array}{l}
n-t \\
k-t
\end{array}\right)
$$

for any $2 \leq t \leq k<n$. By Proposition 6.2 we have

$$
c_{t}\left(\widetilde{\mathcal{R}}_{n, k}^{!}\right)=\frac{c_{t}\left(\mathcal{R}_{n, k}^{!}\right)}{\left(\begin{array}{l}
n \\
k
\end{array}\right)}=\frac{\left(\begin{array}{c}
n-t \\
k-t
\end{array}\right)}{\left(\begin{array}{l}
n \\
k
\end{array}\right)}=\frac{\left(\begin{array}{c}
k \\
t
\end{array}\right)}{\left(\begin{array}{l}
n \\
t
\end{array}\right)} .
$$

8.11. Definition: degrees and ranks of braid group pseudocharacters. By Proposition 8.5, a pseudocharacter $\phi: B_{n} \rightarrow \mathbb{R}$ is uniquely written in the form

$$
\phi=\phi_{n}+\phi_{n-1}+\cdots+\phi_{2},
$$

where $\phi_{k} \in \Gamma_{n, k}$. The number of nontrivial elements on the right-hand side in (13) is called the rank of the pseudocharacter $\phi$. We define the degree of $\phi$ to be the largest $t \in\{2, \ldots, n\}$ with $\phi_{t} \neq 0$.

8.12. Remark. By Remark 8.4 the set of pseudocharacters of degree 1 in $\mathcal{P X}\left(B_{n}\right)$ is empty, and the set of pseudocharacters of degree 2 in $\mathcal{P X}\left(B_{n}\right)$ coincides with the set $\Gamma_{n, 2} \backslash 0$ of nontrivial real-valued homomorphisms.

8.13. Remark. Obviously, the notion of degree introduced in Definition 8.11 generalizes (and agrees with) the notion of degree for subkernel pseudocharacters as described in Definition 8.3

8.14. Remark. Let $\phi: B_{n} \rightarrow \mathbb{R}$ be a pseudocharacter of degree at most $k$. Then for every $\beta \in B_{n}$ the value $\phi(\beta)$ is uniquely determined by the element $\sum_{J \in \mathbf{C}_{n}^{n-k}} \operatorname{RED}_{J}\left(\beta^{n !}\right) \in$ $\mathbb{R} B_{k}$.

8.15. Remark. Given a computational algorithm for a pseudocharacter $\phi: B_{n} \rightarrow \mathbb{R}$, we can construct computational algorithms for its subkernel summands $\phi_{2}, \ldots, \phi_{n}$. Indeed, by definition we have

and

$$
\phi_{n}=\phi-\mathcal{A}_{n, n-1}^{!} \circ \mathcal{I}_{n, n-1}^{!}(\phi)
$$

$$
\phi_{k}=\mathcal{A}_{n, k}^{!} \circ \mathcal{I}_{n, k}^{!}(\phi)-\mathcal{A}_{n, k-1}^{!} \circ \mathcal{I}_{n, k-1}^{!}(\phi) \quad \text { for } \quad k<n .
$$

Obviously, the dimension of the subspace $\langle\phi\rangle \subset \mathcal{P X}\left(B_{n}\right)$ (defined as the subspace generated by the subkernel summands $\left.\phi_{2}, \ldots, \phi_{n}\right)$ is equal to the rank of $\phi$. Our results clearly show that $\langle\phi\rangle$ is the subspace formed by all pseudocharacters in $\mathcal{P X}\left(B_{n}\right)$ that are obtained from $\phi$ by applying various $\Gamma$-diagonal operators (i.e., by applying various compositions and linear combinations of operators belonging to the series $\left\{\mathcal{I}_{n, k}^{!}\right\}_{0 \leq k<n}$, $\left\{\widetilde{\mathcal{R}}_{n, k}^{!}\right\}_{0 \leq k<n},\left\{\mathcal{R}_{n, k}^{!}\right\}_{0 \leq k<n}$, and $\left.\left\{\mathcal{A}_{n, k}^{!}\right\}_{0 \leq k<n}\right)$.

8.16. The space $\mathcal{P} \mathcal{X}_{\infty}\left(B_{\infty}\right)$. In the Introduction it was mentioned that the space $\mathcal{P X}\left(B_{\infty}\right)$ consists of homomorphisms and has dimension 1 . We call a function $\tau: B_{\infty} \rightarrow \mathbb{R}$ a limit pseudocharacter if its restriction to $B_{n}$ is a pseudocharacter for each $n$; the space of all limit pseudocharacters will be denoted by $\mathcal{P} \mathcal{X}_{\infty}\left(B_{\infty}\right)$. The above results immediately imply that there is a natural one-to-one correspondence between the space $\mathcal{P} \mathcal{X}_{\infty}\left(B_{\infty}\right)$ and the space of all infinite sequences $\left\{\phi_{k}\right\}_{k=2,3, \ldots}$ with $\phi_{k} \in \Gamma_{k} \subset \mathcal{P X}\left(B_{k}\right)$, by which we mean that a sequence $\left\{\phi_{k}\right\}_{k=2,3, \ldots}$ corresponds to the limit pseudocharacter $\Phi \in \mathcal{P} \mathcal{X}_{\infty}\left(B_{\infty}\right)$ with the restriction $\left.\Phi\right|_{B_{n}}$ equal for each $n \geq 2$ to the sum

$$
\phi_{n}+\sum_{2 \leq k \leq n-1} \mathcal{A}_{n, k}^{!}\left(\phi_{k}\right) .
$$


8.17. Problem. In [6] it was proved that for each $n \in \mathbb{N}$ the function

$$
\operatorname{sgn}_{n}: B_{n} \rightarrow \mathbb{R}, \quad \operatorname{sgn}_{n}(\beta)=\operatorname{sgn}(L(\beta))
$$

that assigns to a braid $\beta$ the classical signature of the link $L(\beta)$ represented by $\beta$, is a quasimorphism. Let

$$
\overline{\operatorname{sgn}}_{n}: B_{n} \rightarrow \mathbb{R}, \quad \overline{\operatorname{sgn}}_{n}(\beta):=\lim _{k \rightarrow \infty} \frac{\operatorname{sgn}_{n}\left(\beta^{k}\right)}{k}
$$

be the corresponding pseudocharacter (see the Introduction).

Problem. To find the degree and rank of the pseudocharacter $\overline{\operatorname{sgn}}_{n}$ for $n \geq 4$. (It is easy to check that $\overline{\mathrm{ggn}}_{3}$ has degree 3 and rank 2.)

8.18. Problem. From the results of $[6$ and $[8$ it can be deduced that the pseudocharacter $\overline{\operatorname{sgn}}_{3}$ defined in Subsection 8.17 is a linear combination of the twist number (see the definition in [10]) and the exponential sum.

Problem. To find out if there are any other nontrivial linear dependencies among pseudocharacters that can be obtained by $\Gamma$-diagonal operators from the twist numbers and those that can be obtained from the pseudocharacters $\overline{\mathrm{sgn}}_{n}$ defined above.

\section{§9. Properties of the operators $\mathcal{R}_{n, k}^{!}$}

In this section, we prove several properties of the operators $\mathcal{R}_{n, k}^{!}$. In particular, we check that these operators are injective and $\Gamma$-diagonal.

9.1. Lemma. Let $0 \leq k<m<n$. Then

$$
\mathcal{R}_{n, m}^{!} \circ \mathcal{R}_{m, k}^{!}=\left(\begin{array}{c}
n-k \\
m-k
\end{array}\right) \cdot \mathcal{R}_{n, k}^{!},
$$

where $\left(\begin{array}{c}n-k \\ m-k\end{array}\right)=\left(\begin{array}{c}n-k \\ n-m\end{array}\right)=\frac{(n-k) !}{(m-k) !(n-m) !}$.

9.2. Remark. Using Lemma 9.1, Proposition 6.2 and the obvious combinatorial equation $\left(\begin{array}{c}n-k \\ m-k\end{array}\right)=\left(\begin{array}{c}n \\ m\end{array}\right) \cdot\left(\begin{array}{c}m \\ k\end{array}\right) /\left(\begin{array}{c}n \\ k\end{array}\right)$, we see that for the operator $\widetilde{\mathcal{R}}_{n, k}^{!}$(introduced in $\left.\S 3\right)$ we have

$$
\widetilde{\mathcal{R}}_{n, k}^{!}=\widetilde{\mathcal{R}}_{n, m}^{!} \circ \widetilde{\mathcal{R}}_{m, k}^{!}
$$

Proof of Lemma 9.1. We show that

$$
\mathcal{R}_{m, k} \circ \mathcal{R}_{n, m}(\beta)=\frac{\left(\begin{array}{c}
n-k \\
m-k
\end{array}\right)}{m !} \cdot \mathcal{R}_{n, k}\left(\beta^{m !}\right)
$$

for every braid $\beta \in B_{n}$. By definition, for any $0 \leq r<s$ and $\alpha \in B_{s}$, we have

$$
\mathcal{R}_{s, r}(\alpha)=\sum_{J \in \mathbf{C}_{s}^{s-r}} \frac{\operatorname{RED}_{J}\left(\alpha^{s !}\right)}{s !},
$$

where

$$
\mathbf{C}_{s}^{s-r}=\{I \subset\{1, \ldots, s\}:|I|=s-r\}
$$

Consequently,

$$
\begin{aligned}
\mathcal{R}_{m, k} \circ \mathcal{R}_{n, m}(\beta) & =\mathcal{R}_{m, k}\left(\sum_{J \in \mathbf{C}_{n}^{n-m}} \frac{\operatorname{RED}_{J}\left(\beta^{n !}\right)}{n !}\right) \\
& =\frac{1}{n ! m !} \cdot \sum_{I \in \mathbf{C}_{m}^{m-k}} \sum_{J \in \mathbf{C}_{n}^{n-m}} \operatorname{RED}_{I}\left(\left(\operatorname{RED}_{J}\left(\beta^{n !}\right)\right)^{m !}\right) .
\end{aligned}
$$


Since $\mathrm{RED}_{I}$ is a homomorphism from the pure braid group to the pure braid group, for any $J \in \mathbf{C}_{n}^{n-m}$ and $I \in \mathbf{C}_{m}^{m-k}$, we have

$$
\operatorname{RED}_{I}\left(\left(\operatorname{RED}_{J}\left(\beta^{n !}\right)\right)^{m !}\right)=\operatorname{RED}_{I} \circ \operatorname{RED}_{J}\left(\beta^{n ! m !}\right)
$$

Lemma 5.9 easily implies that for any $J \in \mathbf{C}_{n}^{n-m}$ and $I \in \mathbf{C}_{m}^{m-k}$ there exists $K \in \mathbf{C}_{n}^{n-k}$ such that $\mathrm{RED}_{I} \circ \mathrm{RED}_{J} \equiv \mathrm{RED}_{K}$. Furthermore, Lemma [5.9 also implies that for each $K \in \mathbf{C}_{n}^{n-k}$ there are precisely $\left(\begin{array}{c}n-k \\ m-k\end{array}\right)$ pairs $(I, J) \in \mathbf{C}_{m}^{m-k} \times \mathbf{C}_{n}^{n-m}$ such that $\operatorname{RED}_{K} \equiv$ $\mathrm{RED}_{I} \circ \mathrm{RED}_{J}$. This means that

$$
\begin{aligned}
\frac{1}{n ! m !} & \cdot \sum_{I \in \mathbf{C}_{m}^{m-k}} \sum_{J \in \mathbf{C}_{n}^{n-m}} \operatorname{RED}_{I}\left(\left(\operatorname{RED}_{J}\left(\beta^{n !}\right)\right)^{m !}\right) \\
= & \frac{1}{n ! m !} \cdot \sum_{I \in \mathbf{C}_{m}^{m-k}} \sum_{J \in \mathbf{C}_{n}^{n-m}} \operatorname{RED}_{I} \circ \operatorname{RED}_{J}\left(\beta^{n ! m !}\right) \\
& =\frac{\left(\begin{array}{c}
n-k \\
m-k
\end{array}\right)}{n ! m !} \cdot \sum_{K \in \mathbf{C}_{n}^{n-k}} \operatorname{RED}_{K}\left(\beta^{n ! m !}\right) .
\end{aligned}
$$

By the definition of $\mathcal{R}_{n, k}$, we have

$$
\frac{\left(\begin{array}{c}
n-k \\
m-k
\end{array}\right)}{n ! m !} \cdot \sum_{K \in \mathbf{C}_{n}^{n-k}} \operatorname{RED}_{K}\left(\beta^{n ! m !}\right)=\frac{\left(\begin{array}{c}
n-k \\
m-k
\end{array}\right)}{m !} \cdot \mathcal{R}_{n, k}\left(\beta^{m !}\right) .
$$

Now, (14) follows from (15), (16), and (17).

We rewrite (14) in the form

$$
\mathcal{R}_{m, k} \circ \mathcal{R}_{n, m}=\left(\begin{array}{c}
n-k \\
m-k
\end{array}\right) \cdot \mathcal{R}_{n, k} \circ \Lambda_{m !}
$$

where $\Lambda_{m}$ ! is the mapping $B_{n} \rightarrow \mathbb{R} B_{n}$ that sends $\beta \in B_{n}$ to $\frac{\beta^{m !}}{m !}$. It is easily seen that the mapping $\Lambda_{m}$ ! is pseudohomomorphic, and that the induced ring operator $\Lambda_{m !}^{!}$: $\mathcal{P X}\left(B_{n}\right) \rightarrow \mathcal{P X}\left(B_{n}\right)$ is the identity. Therefore,

$$
\begin{aligned}
\mathcal{R}_{n, m}^{!} \circ \mathcal{R}_{m, k}^{!} & =\left(\mathcal{R}_{m, k} \circ \mathcal{R}_{n, m}\right)^{!}=\left(\left(\begin{array}{c}
n-k \\
m-k
\end{array}\right) \cdot \mathcal{R}_{n, k} \circ \Lambda_{m !}\right)^{!} \\
& =\left(\begin{array}{c}
n-k \\
m-k
\end{array}\right) \cdot \Lambda_{m !}^{!} \circ \mathcal{R}_{n, k}^{!}=\left(\begin{array}{c}
n-k \\
m-k
\end{array}\right) \cdot \mathcal{R}_{n, k}^{!} .
\end{aligned}
$$

9.3. Proposition. For any $0 \leq m<n$, the operator $\mathcal{R}_{n, m}^{!}$is $\Gamma$-diagonal and injective.

9.4. Remark. Propositions 9.3 and 6.2 imply that for any $0 \leq m<n$, the operator $\widetilde{\mathcal{R}}_{n, m}^{!}$ is $\Gamma$-diagonal and injective.

Proof of Proposition 9.3. We argue by induction on $n$. The statement is obvious for $n=1$ and $n=2$ because the spaces $\mathcal{P X}\left(B_{0}\right)$ and $\mathcal{P X}\left(B_{1}\right)$ are trivial. 
Suppose that $n \geq 3$ and the operators $\mathcal{R}_{s, t}^{!}$are $\Gamma$-diagonal and injective for any $t<s<n$. Then

$$
\begin{aligned}
& \mathcal{A}_{n, n-1}^{!} \stackrel{1)}{=} \mathcal{R}_{n, n-1}^{!}+\sum_{k=0,1, \ldots, n-2}(-1)^{n-k+1} \cdot \mathcal{R}_{n, k}^{!} \circ \mathcal{I}_{n-1, k}^{!} \\
& \stackrel{2)}{=} \mathcal{R}_{n, n-1}^{!}+\sum_{k=0,1, \ldots, n-2} \frac{(-1)^{n-k+1}}{\left(\begin{array}{c}
n-k \\
1
\end{array}\right)} \cdot \mathcal{R}_{n, n-1}^{!} \circ \mathcal{R}_{n-1, k}^{!} \circ \mathcal{I}_{n-1, k}^{!} \\
& \stackrel{3)}{=} \mathcal{R}_{n, n-1}^{!} \circ \mathcal{X}^{!}, \quad \text { where } \\
& \qquad \mathcal{X}^{!}:=\operatorname{id}_{\mathcal{P X}\left(B_{n-1}\right)}+\sum_{k=0,1, \ldots, n-2} \frac{(-1)^{n-k+1}}{n-k} \cdot \mathcal{R}_{n-1, k}^{!} \circ \mathcal{I}_{n-1, k}^{!} .
\end{aligned}
$$

Indeed, 1) is identity (9) from Definition (7.1, identity 2) is true by Lemma 9.1 and 3) is "factoring out".

By the inductive assumption, the operators $\mathcal{R}_{n-1, k}^{!}$are $\Gamma$-diagonal, so that the operator $\mathcal{X}^{!}$is $\Gamma$-diagonal, being a composition of $\Gamma$-diagonal operators. By Proposition 7.2, the operator $\mathcal{A}_{n, n-1}^{!}$is injective. Since $\mathcal{A}_{n, n-1}^{!}=\mathcal{R}_{n, n-1}^{!} \circ \mathcal{X}^{!}$, the operator $\mathcal{X}^{!}: \mathcal{P X}\left(B_{n-1}\right) \rightarrow \mathcal{P X}\left(B_{n-1}\right)$ is injective. Clearly, a $\Gamma$-diagonal and injective operator $\mathcal{P X}\left(B_{s}\right) \rightarrow \mathcal{P X}\left(B_{s}\right)$ is surjective for any $s$. It follows that $\mathcal{X}^{!}$is one-to-one. On the one hand, the injectivity of $\mathcal{A}_{n, n-1}^{!}$implies the injectivity of $\mathcal{R}_{n, n-1}^{!}$. On the other hand, since $\mathcal{A}_{n, n-1}^{!}$is $\Gamma$-diagonal, so is $\mathcal{R}_{n, n-1}^{!}$. (Obviously, if an operator $X$ is $\Gamma$-diagonal and surjective, and the composition $Y \circ X$ is $\Gamma$-diagonal for an operator $Y$, then $Y$ is $\Gamma$-diagonal.)

Therefore, $\mathcal{R}_{n, n-1}^{!}$is $\Gamma$-diagonal and injective. We also observe that, by Lemma 9.1

$$
\mathcal{R}_{n, m}^{!}=\frac{1}{(n-m) !} \cdot \mathcal{R}_{n, n-1}^{!} \circ \mathcal{R}_{n-1, n-2}^{!} \circ \cdots \circ \mathcal{R}_{m+2, m+1}^{!} \circ \mathcal{R}_{m+1, m}^{!} .
$$

Thus, the operator $\mathcal{R}_{n, m}^{!}$is $\Gamma$-diagonal and injective, being a composition of $\Gamma$-diagonal injective operators.

9.5. Lemma. Let $0<k<n$. Then

$$
\left.\mathcal{I}_{n, k}^{!} \circ \mathcal{R}_{n, k}^{!}\right|_{\Gamma_{k}}=\operatorname{id}_{\Gamma_{k}} .
$$

Proof. It suffices to show that

$$
\left(\mathcal{I}_{n, k}^{!} \circ \mathcal{R}_{n, k}^{!}(\phi)\right)(\beta)=\phi(\beta)
$$

for any $\phi \in \Gamma_{k}$ and $\beta \in B_{k}$. We observe that

$$
\left(\mathcal{I}_{n, k}^{!} \circ \mathcal{R}_{n, k}^{!}(\phi)\right)(\beta)=\phi\left(\mathcal{R}_{n, k} \circ \mathcal{I}_{n, k}(\beta)\right)=\phi\left(\mathcal{R}_{n, k}(\beta)\right)
$$

(see Convention 1.3). By the definition of the mapping $\mathcal{R}_{n, k}$, we have

$$
\phi\left(\mathcal{R}_{n, k}(\beta)\right)=\sum_{J \in \mathbf{C}_{n}^{n-k}} \frac{\phi\left(\operatorname{RED}_{J}\left(\beta^{n !}\right)\right)}{n !} .
$$

For $J \in \mathbf{C}_{n}^{n-k}$, we put $J_{1}:=J \cap\{1, \ldots, k\}$ and $J_{2}:=J \backslash J_{1}$. Then $J_{1} \cup J_{2}=J$, $J_{1} / J_{2}=J_{1}$ (see the notation in Subsection [5.2), $J_{2} \subset\{k+1, k+2, \ldots\}$, and $\beta^{n !} \in B_{k}$.

By Lemmas 5.9 and 5.7 these relations imply that

$$
\operatorname{RED}_{J}\left(\beta^{n !}\right)=\operatorname{RED}_{J_{1} \cup J_{2}}\left(\beta^{n !}\right) \stackrel{[5.9}{=} \operatorname{RED}_{J_{1} / J_{2}} \circ \operatorname{RED}_{J_{2}}\left(\beta^{n !}\right) \stackrel{[5.7}{=} \operatorname{RED}_{J_{1} / J_{2}}\left(\beta^{n !}\right)=\operatorname{RED}_{J_{1}}\left(\beta^{n !}\right) \text {. }
$$

By Lemma [5.6. $\operatorname{RED}_{J_{1}}\left(\beta^{n !}\right) \in B_{k-\left|J_{1}\right|}$. Consequently, since $\phi \in \Gamma_{k}$, in the case where $J_{1} \neq \varnothing$ we get

$$
\phi\left(\operatorname{RED}_{J}\left(\beta^{n !}\right)\right)=\phi\left(\operatorname{RED}_{J_{1}}\left(\beta^{n !}\right)\right)=0 .
$$


Clearly, the condition $J_{1}=\varnothing$ implies that $J=\{k+1, \ldots, n\}$. Therefore,

$$
\sum_{J \in \mathbf{C}_{n}^{n-k}} \frac{\phi\left(\operatorname{RED}_{J}\left(\beta^{n !}\right)\right)}{n !}=\frac{\phi\left(\operatorname{RED}_{\{k+1, \ldots, n\}}\left(\beta^{n !}\right)\right)}{n !} .
$$

The definition of pseudocharacters and Lemma 5.7 show that

$$
\frac{\phi\left(\operatorname{RED}_{\{k+1, \ldots, n\}}\left(\beta^{n !}\right)\right)}{n !} \stackrel{5.7}{=} \frac{\phi\left(\beta^{n !}\right)}{n !}=\phi(\beta) .
$$

The required identity follows from the chain of identities (21), (22), (23), and (24).

\section{ACKNOWLEDGMENTS}

The author is cordially grateful to S. S. Podkorytov for valuable remarks and useful discussions.

\section{REFERENCES}

[1] R. Bell and D. Margalit, Injections of Artin groups, Comment. Math. Helv. 82 (2007), no. 4, 725-751. MR2341838 (2009b:20062)

[2] M. Bestvina and K. Fujiwara, Bounded cohomology of subgroups of mapping class groups, Geom. Topol. 6 (2002), 69-89. MR1914565 (2003f:57003)

[3] K. S. Brown, Cohomology of groups, Grad. Texts in Math., vol. 87, Springer-Verlag, New YorkBerlin, 1982. MR.0672956 (83k:20002)

[4] J. Dyer and E. Grossman, The automorphism groups of the braid groups, Amer. J. Math. 103 (1981), no. 6, 1151-1169. MR0636956 (82m:20041)

[5] J.-M. Gambaudo and É. Ghys, Commutators and diffeomorphisms of surfaces, Ergodic Theory Dynam. Systems 24 (2004), no. 5, 1591-1617. MR2104597 (2006d:37071)

[6] , Braids and signatures, Bull. Soc. Math. France 133 (2005), no. 4, 541-579. MR2233695 (2007d:57014)

[7] K. Honda, W. Kazez, and G. Matić, Right-veering diffeomorphisms of compact surfaces with boundary. I, Invent. Math. 169 (2007), no. 2, 427-449. MR2318562 (2008e:57028)

[8] ㄴ. Right-veering diffeomorphisms of compact surfaces with boundary. II, Geom. Topol. 12 (2008), no. 4, 2057-2094. MR2431016 (2009i:57057)

[9] V. Lin, Braids and permutations, Preprint, ArXiv:math/0404528.

[10] A. V. Malyutin, Twist number of (closed) braids, Algebra i Analiz 16 (2004), no. 5, 59-91; English transl., St. Petersburg Math. J. 16 (2005), no. 5, 791-813. MR2106667 (2005j:57011)

[11] _ Pseudocharacters of braid groups and prime links, Algebra i Analiz 21 (2009), no. 2, 113-135; English transl. in St. Petersburg Math. J. 21 (2010), no. 2 (the previous article in this issue).

[12] A. A. Markov, Foundations of the algebraic theory of braids, Trudy Mat. Inst. Steklov. 16 (1945), 3-53. (Russian) MR0017279 (8:131c)

St. Petersburg Branch, Steklov Institute of Mathematics, Russian Academy of Sciences, Fontanka 27, St. Petersburg 191023, Russia

E-mail address: malyutin@pdmi.ras.ru

Received 16/SEP/2008

Translated by THE AUTHOR 